\title{
The unknotting number and classical invariants, I
}

\author{
MACIEJ BORODZIK \\ STEFAN FRIEDL
}

\begin{abstract}
Given a knot $K$ we introduce a new invariant coming from the Blanchfield pairing and we show that it gives a lower bound on the unknotting number of $K$. This lower bound subsumes the lower bounds given by the Levine-Tristram signatures, by the Nakanishi index and it also subsumes the Lickorish obstruction to the unknotting number being equal to one. Our approach in particular allows us to show for 25 knots with up to 12 crossings that their unknotting number is at least three, most of which are very difficult to treat otherwise.
\end{abstract}

$57 \mathrm{M} 27$

\section{Introduction}

Let $K \subset S^{3}$ be a knot. Throughout this paper a knot is always assumed to be oriented. A crossing change is one of the two local moves on a knot diagram given in Figure 1.
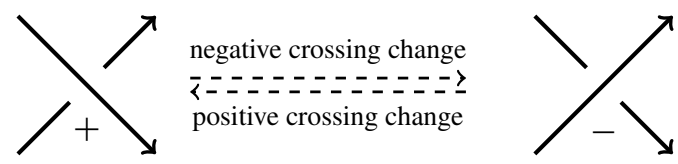

Figure 1: Negative and positive crossing change

The unknotting number $u(K)$ of a knot $K$ is defined to be the minimal number of crossing changes necessary to turn $K$ into the unknot. The unknotting number is one of the most elementary invariants of a knot, but also one of the most intractable. Whereas upper bounds can be found readily using diagrams, it is much harder to find nontrivial lower bounds.

In this paper we will for the most part study a closely related invariant, namely the algebraic unknotting number $u_{a}(K)$, which is defined to be the minimal number of crossing changes necessary to turn $K$ into a knot with trivial Alexander polynomial. By Fogel [13] and Saeki [60] this is equivalent to the original definition by Murakami [48] given in terms of "algebraic unknotting moves" on Seifert matrices. It is clear that $u(K) \geq u_{a}(K)$, and that in general this is not an equality. For example for any nontrivial knot $K$ with trivial Alexander polynomial we have $u(K) \geq 1$ and $u_{a}(K)=0$. 


\subsection{Review of classical invariants}

Let us first fix some terminology. Let $F$ be a Seifert surface for $K$ and let $v_{1}, \ldots, v_{2 n}$ be a collection of embedded simple closed curves on $F$ which represent a basis for $H_{1}(F ; \mathbb{Z})$. The corresponding Seifert matrix $V$ is defined as the matrix with $(i, j)-$ entry given by

$$
\operatorname{lk}\left(v_{i}, v_{j}^{+}\right),
$$

where we denote by $v_{j}^{+}$the positive pushoff of $v_{j}$. The $S$-equivalence class of the Seifert matrix is well known to be an invariant of $K$ (see Lickorish [43, Theorem 8.4] for details). The $S$-equivalence class of the Seifert matrix will be denoted by $V=V_{K}$. By abuse of notation we will often denote by $V=V_{K}$ a representative of the $S$ equivalence class. In this paper, by a classical invariant of a knot we mean an invariant which is determined by $V_{K}$.

Given a knot $K$ we denote by $X(K)=S^{3} \backslash v K$ the exterior of $K$ and we denote by $\Sigma(K)$ its branched cover. We now give several well-known examples of classical invariants which will play a role in the paper (in the following the matrix $V$ is a $2 n \times 2 n$ Seifert matrix for $K$ ):

(1) The Alexander polynomial is defined as

$$
\Delta_{K}(t)=t^{-n} \cdot \operatorname{det}\left(V t-V^{t}\right) \in \mathbb{Z}\left[t^{ \pm 1}\right]
$$

(note that $\Delta_{K}(t)$ is well defined with no indeterminacy, and $\Delta_{K}(1)=1$ ).

(2) The knot determinant $\operatorname{det}(K)=(-1)^{n} \operatorname{det}\left(V+V^{t}\right)$, which in this paper is viewed as a signed invariant.

(3) The isometry type of the linking pairing

$$
l(K): H_{1}(\Sigma(K) ; \mathbb{Z}) \times H_{1}(\Sigma(K) ; \mathbb{Z}) \rightarrow \mathbb{Q} / \mathbb{Z},
$$

which is isometric to the pairing

$$
\mathbb{Z}^{2 n} /\left(V+V^{t}\right) \mathbb{Z}^{2 n} \times \mathbb{Z}^{2 n} /\left(V+V^{t}\right) \mathbb{Z}^{2 n} \rightarrow \mathbb{Q} / \mathbb{Z}, \quad(v, w) \mapsto v^{t}\left(V+V^{t}\right)^{-1} w
$$

(we refer to Gordon [17] for details).

(4) The Blanchfield pairing

$$
\lambda(K): H_{1}\left(X(K) ; \mathbb{Z}\left[t^{ \pm 1}\right]\right) \times H_{1}\left(X(K) ; \mathbb{Z}\left[t^{ \pm 1}\right]\right) \rightarrow \mathbb{Q}(t) / \mathbb{Z}\left[t^{ \pm 1}\right]
$$

is a hermitian nonsingular pairing on the Alexander module $H_{1}\left(X(K) ; \mathbb{Z}\left[t^{ \pm 1}\right]\right)$ (we refer to Section 2.2 for the definition).

(5) The Nakanishi index $m(K)$, ie the minimal number of generators of the Alexander module $H_{1}\left(X(K) ; \mathbb{Z}\left[t^{ \pm 1}\right]\right)$. 
(6) Given $z \in S^{1}$ the Levine-Tristram signature is defined as

$$
\sigma_{z}(K)=\operatorname{sign}\left(V(1-z)+V^{t}\left(1-z^{-1}\right)\right)
$$

(note that $\sigma_{-1}(K)$ is just the ordinary knot signature $\sigma(K)$ ).

(7) Given $z \in \mathbb{C} \backslash\{0,1\}$ the nullity is defined as

$$
\eta_{z}(K)=\operatorname{null}\left(V(1-z)+V^{t}\left(1-z^{-1}\right)\right) ;
$$

furthermore $\eta_{1}(K)$ is by convention defined to be 0 .

\subsection{Definition of the invariant $n(K)$}

Given a hermitian $n \times n$-matrix $A$ over $\mathbb{Z}\left[t^{ \pm 1}\right]$ with $\operatorname{det}(A) \neq 0$ we denote by $\lambda(A)$ the pairing

$\lambda(A): \mathbb{Z}\left[t^{ \pm 1}\right]^{n} / A \mathbb{Z}\left[t^{ \pm 1}\right]^{n} \times \mathbb{Z}\left[t^{ \pm 1}\right]^{n} / A \mathbb{Z}\left[t^{ \pm 1}\right]^{n} \rightarrow \mathbb{Q}(t) / \mathbb{Z}\left[t^{ \pm 1}\right], \quad(a, b) \mapsto \bar{a}^{t} A^{-1} b$,

where we view $a, b$ as represented by column vectors in $\mathbb{Z}\left[t^{ \pm 1}\right]^{n}$. Note that $\lambda(A)$ is a nonsingular, hermitian pairing.

Let $K$ be a knot. We define $n(K)$ to be the minimal size of a hermitian matrix $A$ over $\mathbb{Z}\left[t^{ \pm 1}\right]$ such that

- $\lambda(A) \cong \lambda(K)$, ie $\lambda(A)$ is isometric to the Blanchfield pairing of $K$,

- the matrix $A(1)$ is congruent over $\mathbb{Z}$ to a diagonal matrix which has \pm 1 on the diagonal.

In Section 2.2 we will see that the Blanchfield pairing of $K$ can indeed be represented by such a matrix $A$, ie we will show that $n(K)$ is actually defined. We will furthermore show that $n(K) \leq \operatorname{deg} \Delta_{K}(t)+1$. Note that $n(K)=0$ if and only if the Alexander polynomial of $K$ is trivial. Finally note that $n(K)$ is a classical invariant since the Blanchfield pairing is a classical invariant.

\subsection{Classical bounds for the unknotting number}

We will now quickly summarize all previous classical lower bounds on the unknotting number which are known to the authors.

The first lower bounds on the unknotting number go back to Wendt [71]; they are subsumed by the following inequality due to Nakanishi [50]:

$$
u(K) \geq m(K) .
$$

We discuss it in Section 4.1. 
It has been known since the work of Murasugi [49] that Levine-Tristram signatures give rise to lower bounds on the unknotting number. In particular the following inequality holds:

$u_{a}(K) \geq \mu(K):=\frac{1}{2}\left(\max \left\{\eta_{z}(K)+\sigma_{z}(K) \mid z \in S^{1}\right\}+\max \left\{\eta_{z}(K)-\sigma_{z}(K) \mid z \in S^{1}\right\}\right)$

This inequality is in all likelihood known to the experts, but we are not aware of a reference and we thus give a proof (together with a more refined statement) in Section 4.2.

By Saeki [60, Proposition 4.1] the topological 4-ball genus $g_{4}^{\text {top }}(K)$ is a lower bound on the algebraic unknotting number $u_{a}(K)$. Livingston [44] introduced a classical invariant $\rho(K)$ which gives a lower bound on $g_{4}^{\text {top }}(K)$. In Section 4.3 we will slightly modify Livingston's invariant to define a new classical invariant $\rho_{\mathbb{Z}\left[t^{ \pm 1}\right]}(K)$ which satisfies

$$
g_{4}^{\text {top }}(K) \geq \rho_{\mathbb{Z}\left[t^{ \pm 1}\right]}(K) \geq \rho(K)
$$

and we will show that $n(K) \geq \rho_{\mathbb{Z}\left[t^{ \pm 1}\right]}(K)$.

We now recall several classical obstructions to a knot $K$ having "small" algebraic unknotting number. If $K$ can be unknotted using a single $\epsilon-$ crossing change (with $\epsilon \epsilon$ $\{-1,1\})$, then by the work of Lickorish [42] there exists a generator $h$ of $H_{1}(\Sigma(K) ; \mathbb{Z})$ such that

$$
l(h, h)=\frac{-2 \epsilon}{\operatorname{det}(K)} \in \mathbb{Q} / \mathbb{Z} .
$$

Recently Jabuka [23] also introduced an obstruction to the unknotting number being one. In Section 4.6 we will see that it is subsumed by the Lickorish obstruction. Also note that the Lickorish obstruction was generalized by Fogel, Murakami and Rickard (see $[13 ; 48]$, respectively) in terms of the Blanchfield pairing. Finally note that if $|\sigma(K)|=4$, then Stoimenow [64, Proposition 5.2] gives a classical obstruction to $u_{a}(K)=2$ in terms of the determinant of $K$. To the best of our knowledge the above is a complete list of lower bounds on the unknotting number given by classical invariants.

Remark (1) Lower bounds on the unknotting number have also been obtained using gauge theory (see Cochran and Lickorish [10] and Kronheimer and Mrowka [37]), Khovanov homology (see Rasmussen [59]) and Heegaard-Floer homology (see Rasmussen [58], Ozsváth and Szabó [54; 55], Owens [51], Greene [19] and Sarkar [61]) and various other methods (see Kanenobu and Murakami [24], Kobayashi [36], Scharlemann and Thompson [63], Miyazawa [46], Traczyk [67], Stoimenow [64], Ma and Qiu [45] and Gordon and Luecke [18]). Note though, 
that with the exception of the Rasmussen $s$-invariant, the Ozsváth-Szabo $\tau-$ invariant and the Owens obstruction most of the above are in fact obstructions to the unknotting number being equal to one or two.

(2) Without doubt, the most important result on unknotting numbers has been the resolution of the Milnor conjecture by Kronheimer and Mrowka [37]: the unknotting number of the $(p, q)$-torus knot equals $(p-1)(q-1) / 2$. We also refer to $[54 ; 59 ; 61]$ for alternative proofs. Finally we refer to Boileau and Weber [3] for an interesting pregauge theory discussion of the problem.

\subsection{The main theorem}

Our main theorem is the following.

Theorem 1.1 Let $K$ be a knot which can be turned into an Alexander polynomial one knot using $u_{+}$positive crossing changes and $u_{-}$negative crossing changes. Then there exists a hermitian matrix $A(t)$ of size $u_{+}+u_{-}$over $\mathbb{Z}\left[t^{ \pm 1}\right]$ with the following two properties:

(1) $\lambda(A(t)) \cong \lambda(K)$.

(2) $A(1)$ is a diagonal matrix such that $u_{+}$diagonal entries are equal to -1 and $u_{-}$ diagonal entries are equal to 1 .

In particular $u_{a}(K) \geq n(K)$.

In Section 4 we will show that the lower bound on the algebraic unknotting number from Theorem 1.1 contains, to the best knowledge of the authors, all previous classical lower bounds to the unknotting number. This result can be summarized in the following theorem:

Theorem 1.2 The invariant $n(K)$ subsumes the following unknotting obstructions:

(1) The Nakanishi index (see Section 4.1).

(2) The invariant $\mu(K)$ (see Section 4.2).

(3) $\rho_{\mathbb{Z}\left[t^{ \pm 1}\right]}(K)$ and in particular Livingston's invariant $\rho(K)$ (see Section 4.3).

(4) The Fogel-Murakami-Rickard obstruction (see Section 4.4).

(5) The Lickorish obstruction and the Jabuka obstruction (see Sections 4.5 and 4.6).

(6) The Stoimenow obstruction (see Section 4.7).

In particular all of the above give lower bounds on the algebraic unknotting number. 
The precise statements and the proofs are given in the indicated parts of Section 4 .

Remark The fact that $n(K)$ subsumes all other classical lower bounds does not invalidate those earlier bounds, since all but the first one are directly computable, whereas at the moment there is no algorithm to calculate $n(K)$ in general.

Remark The fact that the earlier classical lower bounds on the unknotting number give in fact lower bounds on the algebraic unknotting number can also at times be deduced from reading carefully the original proofs.

Fogel [14] proved the following remarkable partial converse to Theorem 1.1.

Theorem 1.3 If $n(K)=1$, then $u_{a}(K)=1$.

Fogel's proof is constructive in the sense that in many cases, given a knot $K$ with $n(K)=1$, one can actually find explicitly a diagram and a crossing change which turns $K$ into an Alexander polynomial one knot. We refer to [13, Section 3; 14, Section 4] for more details. The results of Fogel make plausible the following conjecture.

Conjecture 1.4 For any knot $K$ we have

$$
n(K)=u_{a}(K)
$$

We plan to investigate this conjecture in a future paper.

Added in proof In [5] we provide a proof for Conjecture 1.4.

\subsection{Diagrammatic comparison of classical invariants}

In order to show how the newly defined invariant $n(K)$ fits into the bigger picture of knot invariants, we present in Figure 2 a diagram which shows the relationship between various topological and classical invariants. Beyond the invariants introduced above we will also use the topological invariants

$g_{3}(K)=$ minimal genus of an orientable surface in $S^{3}$ cobounding the knot $K$, $g_{4}^{\text {smooth }}(K)=$ minimal genus of an orientable smooth surface in $D^{4}$ cobounding the knot $K$, $g_{4}^{\text {top }}(K)=$ minimal genus of a locally flat surface in $D^{4}$ cobounding the knot $K$, 
and the classical invariants

$$
\eta(K):=\max \left\{\eta_{z}(K) \mid z \in \mathbb{C} \backslash\{0\}\right\},
$$

$m_{\mathbb{R}}(K):=$ minimal number of generators of $H_{1}\left(X(K) ; \mathbb{R}\left[t^{ \pm 1}\right]\right)$,

$n_{\mathbb{R}}(K):=$ the invariant $n(K)$ defined over real numbers, ie the minimal size of a hermitian matrix over $\mathbb{R}\left[t^{ \pm 1}\right]$ representing the Blanchfield pairing over $\mathbb{R}, H_{1}\left(X(K) ; \mathbb{R}\left[t^{ \pm 1}\right]\right) \times H_{1}\left(X(K) ; \mathbb{R}\left[t^{ \pm 1}\right]\right) \rightarrow \mathbb{R}(t) / \mathbb{R}\left[t^{ \pm 1}\right]$.

It is straightforward to see that $m_{\mathbb{R}}(K)=\eta(K)$. In a future paper [6] we will show that furthermore

$$
n_{\mathbb{R}}(K)=\max \{\mu(K), \eta(K)\}
$$

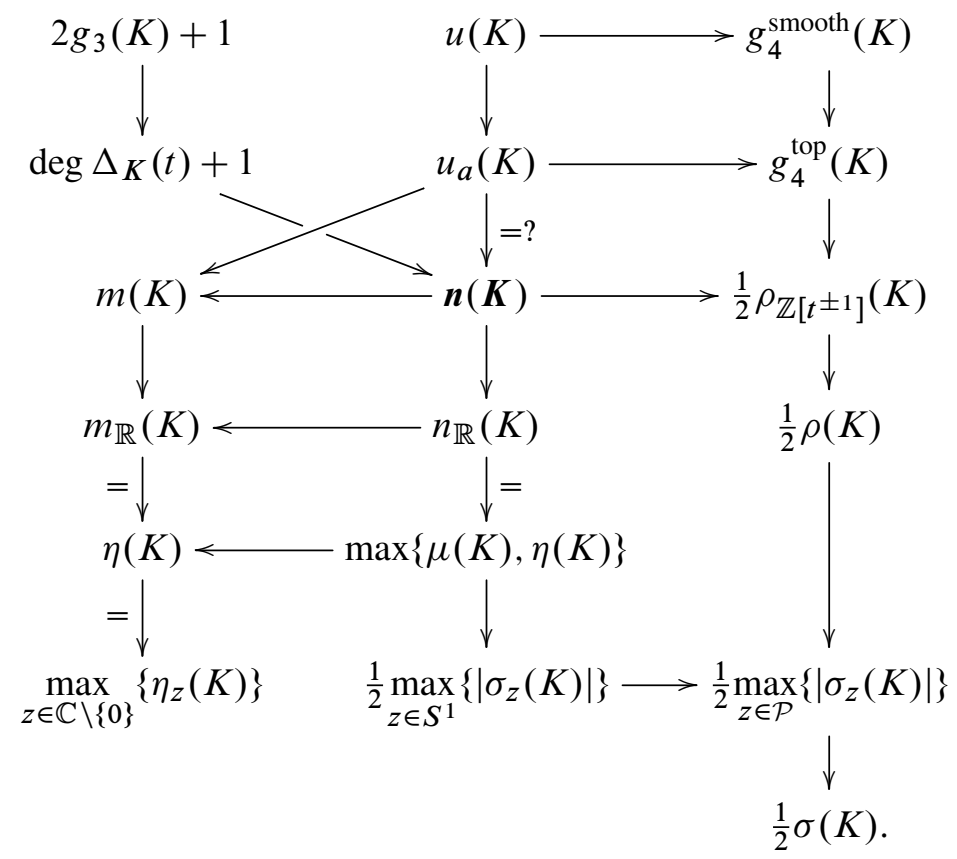

Figure 2: Diagrammatic summary of known invariants: here $\mathcal{P}$ denotes the set of all prime power roots of unity. We use the following notation: given two knot invariants $f$ and $g$ we write $f(K) \rightarrow g(K)$ if $f(K) \geq g(K)$ for every knot $K$. The existence of an arrow is in all cases either well known, or a tautology or it follows from the results in this section. If two invariants are not related by a concatenation of arrows, then in most cases it is known that they are unrelated. If an arrow is not decorated by an $=$ sign, then in most cases it is known that the invariants are indeed not equal. 


\subsection{Applications and examples}

Our understanding of the relation between the $n(K)$ invariant and the presentation matrix for the linking pairing of the double branched cover (see Section 3, especially Lemma 3.3) allows us to provide new computable obstructions for $u(K)=2$ and $u(K)=3$, which are related to Owens' obstruction from [51]. The idea behind the results in Sections 5.2 and 5.3 is the following. If the Blanchfield pairing can be realized by an $n \times n$ matrix over $\mathbb{Z}\left[t^{ \pm}\right]$, then there exists an $n \times n$ integer matrix of a certain type which represents the linking pairing $l(K)$ of the double branched cover. Up to congruence there exist finitely many such matrices, which furthermore in many cases can be listed explicitly. It is then straightforward to verify whether or not $l(K)$ can be represented by any of these matrices.

Among knots with up to 12 crossings there are 25 knots with $m(K) \leq 2$ and $\mu(K) \leq 4$, but for which our approach shows that $n(K) \geq 3$. Out of these 25 knots the Stoimenow obstruction detects four knots. To the best of our knowledge no other classical obstruction applies to these 25 knots. Also, in most cases the Rasmussen $s$-invariant and the Ozsváth-Szabó $\tau$-invariant cannot detect the unknotting number. We furthermore checked the $u(K)=3$ obstruction for all knots with up to 14 crossings with $|\sigma(K)|=6$ and $m(K) \leq 3$. We found that it applies precisely to two such knots, namely $14 n_{12777}$ and $14 a_{4637}$. We have not yet implemented the obstruction to $u(K)=n$ for higher values of $n$.

Our new obstruction to $n(K)=2$ now allows us to completely determine the algebraic unknotting number for all knots with up to 11 crossings. Details are given in Section 6 and by the authors in [4].

Acknowledgments This paper greatly benefitted from conversations with Baskar Balasubramanyam, Slaven Jabuka, Raymond Lickorish, Brendan Owens, Andrew Ranicki, András Stipsicz and Alexander Stoimenow. We would like to thank Micah Fogel for sending us his thesis and Hitoshi Murakami for supplying us with a copy of [48]. The website knotinfo [9], which is maintained by Jae Choon Cha and Chuck Livingston, has been an invaluable tool for finding examples and testing our algorithms. We are also very grateful that Alexander Stoimenow provided us with braid descriptions for knots up to 14 crossings and we wish to thank Julia Collins for help with obtaining Seifert matrices from the braid descriptions. The paper also greatly benefited from a long list of helpful comments by the referee.

Finally we also would like to express our gratitude to the Renyi Institute for its hospitality and to the London Mathematical Society for a travel grant.

The first author is supported by Polish MNiSzW grant number N N201 397937 and also by the Foundation for Polish Science FNP. 


\section{Proof of Theorem 1.1}

Throughout Section 2 we write

$$
\Lambda:=\mathbb{Z}\left[t^{ \pm 1}\right] \text { and } \Omega:=\mathbb{Q}(t) .
$$

As usual we also identify $\Lambda$ with the group ring of $\mathbb{Z}$.

\subsection{Poincaré duality and the universal coefficient spectral sequence}

In this section we will collect several facts which we will use continuously throughout the paper.

Throughout the paper $X$ will always denote a manifold whose first homology group is isomorphic to $\mathbb{Z}$. We denote the infinite cyclic covering of $X$ by $\pi: \hat{X} \rightarrow X$. Given a submanifold $Y \subset X$ we write $\hat{Y}=\pi^{-1}(Y)$. Note that $\mathbb{Z}$ is the deck transformation group of $\hat{X}$. This defines a canonical action of $\Lambda=\mathbb{Z}[\mathbb{Z}]$ on $C_{*}(\hat{X}, \hat{Y} ; \mathbb{Z})$. Given any $\Lambda$-module $N$ we now define

$$
\begin{aligned}
H^{*}(X, Y ; N) & :=H_{*}\left(\operatorname{Hom}_{\Lambda}\left(C_{*}(\hat{X}, \hat{Y} ; \mathbb{Z}), N\right)\right), \\
H_{*}(X, Y ; N) & :=H_{*}\left(\overline{C_{*}(\hat{X}, \hat{Y} ; \mathbb{Z})} \otimes_{\Lambda} N\right) .
\end{aligned}
$$

(Here, and throughout the paper, given a module $H$ over $\Lambda$ we denote by $\bar{H}$ the module with the involuted $\Lambda$-structure, ie $\bar{H}=H$ as abelian groups, but multiplication by $p(t) \in \Lambda$ in $\bar{H}$ is the same as multiplication by $\overline{p(t)}=p\left(t^{-1}\right)$ in $H$.) In particular we can consider the modules $H_{*}(X, Y ; \Lambda), H^{*}(X, Y ; \Lambda), H_{*}(X, Y ; \Omega)$ and $H^{*}(X, Y ; \Omega)$. When $Y=\varnothing$, then we will suppress $Y$ from the notation.

Note that the quotient field $\Omega$ is flat over the ring $\Lambda$. In particular, $H_{*}(X, Y ; \Omega) \cong$ $H_{*}(X, Y ; \Lambda) \otimes_{\Lambda} \Omega$ and $H^{*}(X, Y ; \Omega) \cong H^{*}(X, Y ; \Lambda) \otimes_{\Lambda} \Omega$.

Suppose that $X$ is an $n$-manifold; then for any $\Lambda$-module $N$ Poincaré duality defines isomorphisms of $\Lambda$-modules

$$
\begin{aligned}
H_{i}(X, \partial X ; N) & \cong \overline{H^{n-i}(X ; N)}, \\
H_{i}(X ; N) & \cong \overline{H^{n-i}(X, \partial X ; N)} .
\end{aligned}
$$

Finally we recall the universal coefficient spectral sequence (UCSS); we refer to Levine [41, Theorem 2.3] and Hillman [20, Section 2.1] for details. Let $N$ be any $\Lambda$-module. Then the UCSS starts with $E_{2}^{p, q}=\operatorname{Ext}_{\Lambda}^{q}\left(H_{p}(X ; \Lambda), N\right)$ and converges to $H^{*}(X ; N)$. The differentials at the $r$-stage of this sequence have degree $(1-r, r)$. 
Note that for any two $\Lambda$-modules $H$ and $N$ the module $\operatorname{Ext}_{\Lambda}^{0}(H, N)$ is canonically isomorphic to $\operatorname{Hom}_{\Lambda}(H, N)$. Also note that

$$
\operatorname{Ext}_{\Lambda}^{p}(H, N)=0
$$

for any $p>2$ since $\Lambda$ has global dimension 2 . Finally note that $\mathbb{Z}$, viewed as a $\mathbb{Z}\left[t^{ \pm 1}\right]$-module with trivial $t$-action, admits a free resolution of length 1 . It now follows that $\operatorname{Ext}_{\Lambda}^{p}(\mathbb{Z}, N)=0$ for any $p>1$.

\subsection{Seifert matrices and Blanchfield pairings}

Let $K \subset S^{3}$ be a knot. We consider the following sequence of maps:

$$
\begin{aligned}
\Phi: H_{1}(X(K) ; \Lambda) & \rightarrow H_{1}(X(K), \partial X(K) ; \Lambda) \\
& \rightarrow \overline{H^{2}(X(K) ; \Lambda) \cong} \cong \overline{H^{1}(X(K) ; \Omega / \Lambda)} \\
& \rightarrow \overline{\operatorname{Hom}_{\Lambda}\left(H_{1}(X(K) ; \Lambda), \Omega / \Lambda\right)} .
\end{aligned}
$$

Here the first map is the inclusion induced map, the second map is Poincaré duality, the third map comes from the long exact sequence in cohomology corresponding to the coefficients $0 \rightarrow \Lambda \rightarrow \Omega \rightarrow \Omega / \Lambda \rightarrow 0$, and the last map is the evaluation map. It is well known that the first map is an isomorphism, the second map is obviously an isomorphism, and it follows from the UCSS (and the straightforward calculation that $\operatorname{Ext}_{\Lambda}^{p}(\mathbb{Z}, \Omega / \Lambda)=0$ for $\left.p \geq 1\right)$ that the evaluation map is also an isomorphism. It follows that the above maps thus define a nonsingular pairing

$$
\begin{aligned}
\lambda(K): H_{1}(X(K) ; \Lambda) \times H_{1}(X(K) ; \Lambda) & \rightarrow \Omega / \Lambda, \\
(a, b) & \mapsto \Phi(a)(b),
\end{aligned}
$$

called the Blanchfield pairing of $K$. This pairing is well known to be hermitian; in particular $\lambda(K)\left(a_{1}, a_{2}\right)=\overline{\lambda(K)\left(a_{2}, a_{1}\right)}$ and $\lambda(K)\left(\mu_{1} a_{1}, \mu_{2} a_{2}\right)=\bar{\mu}_{1} \lambda(K)\left(a_{1}, a_{2}\right) \mu_{2}$ for $\mu_{i} \in \Lambda, a_{i} \in H_{1}(X(K) ; \Lambda)$. We refer to Blanchfield [2] for an alternative definition and for further details.

Remark The Alexander polynomial $\Delta_{K}(t)$ is well known to annihilate the Alexander module. It now follows easily from the definition of $\lambda(K)$ that $\lambda(K)$ takes values in $\Delta_{K}(t)^{-1} \mathbb{Z}\left[t^{ \pm 1}\right] / \mathbb{Z}\left[t^{ \pm 1}\right] \subset \mathbb{Q}(t) / \mathbb{Z}\left[t^{ \pm 1}\right]$. This fact also follows from the description of the Blanchfield pairing in terms of Seifert matrices due to Kearton [27, Section 8] which we recall below.

Let $V$ be any matrix of size $2 k$ which is $S$-equivalent to a Seifert matrix for $K$. Note that $V-V^{t}$ is antisymmetric and it satisfies $\operatorname{det}\left(V-V^{t}\right)=(-1)^{k}$. It is well known 
that, possibly after replacing $V$ by $P V P^{t}$ for an appropriate $P$, we have

$$
V-V^{t}=\left(\begin{array}{cc}
0 & \mathrm{id}_{k} \\
-\mathrm{id}_{k} & 0
\end{array}\right)
$$

Following Ko [35, Section 4] we now define $A_{K}(t)$ to be the matrix

$$
\begin{aligned}
\left(\begin{array}{cc}
\left(1-t^{-1}\right)^{-1} \mathrm{id}_{k} & 0 \\
0 & \mathrm{id}_{k}
\end{array}\right) V\left(\begin{array}{cc}
\mathrm{id}_{k} & 0 \\
0 & (1-t) \mathrm{id}_{k}
\end{array}\right) \\
+\left(\begin{array}{cc}
\mathrm{id}_{k} & 0 \\
0 & \left(1-t^{-1}\right) \mathrm{id}_{k}
\end{array}\right) V^{t}\left(\begin{array}{cc}
(1-t)^{-1} \mathrm{id}_{k} & 0 \\
0 & \mathrm{id}_{k}
\end{array}\right)
\end{aligned}
$$

Note that the matrix $A_{K}(t)$ is a hermitian matrix defined over $\Lambda$ and note that $\operatorname{det}\left(A_{K}(1)\right)=(-1)^{k}$ (see [35]). Also note that

$$
\left(\begin{array}{cc}
\left(1-t^{-1}\right) \mathrm{id}_{k} & 0 \\
0 & \operatorname{id}_{k}
\end{array}\right) A_{K}(t)\left(\begin{array}{cc}
(1-t) \mathrm{id}_{k} & 0 \\
0 & \operatorname{id}_{k}
\end{array}\right)=(1-t) V+\left(1-t^{-1}\right) V^{t}
$$

We now have the following proposition.

Proposition 2.1 Let $K$ be a knot and $A_{K}(t)$ as above; then $\lambda\left(A_{K}(t)\right) \cong \lambda(K)$.

Note that the isometry type of the Blanchfield pairing in fact determines the $S$ equivalence class of the Seifert matrix; see Trotter [69] and Ranicki [57]. In that sense the Blanchfield pairing is a "complete" classical invariant, ie it determines all other classical invariants.

Proof First note that the Blanchfield pairing $\lambda(K)$ is isometric to the following pairing (we refer to [27, Section 8] for details):

$$
\Lambda^{2 k} /\left(V t-V^{t}\right) \Lambda^{2 k} \times \Lambda^{2 k} /\left(V t-V^{t}\right) \Lambda^{2 k} \stackrel{(t-1)\left(V t-V^{t}\right)^{-1}}{\longrightarrow} \Omega / \Lambda .
$$

The notation we use here, and similarly below, means that to $a, b \in \Lambda^{2 k}$ we associate $\bar{a}^{t}(t-1)\left(V t-V^{t}\right)^{-1} b$. We now write $\Lambda_{0}:=\mathbb{Z}\left[t, t^{-1},(1-t)^{-1}\right]$ and we let

$$
P:=\left(\begin{array}{cc}
t^{-1} \mathrm{id}_{k} & 0 \\
0 & (t-1)^{-1} \mathrm{id}_{k}
\end{array}\right)
$$


We consider the following commutative diagram:

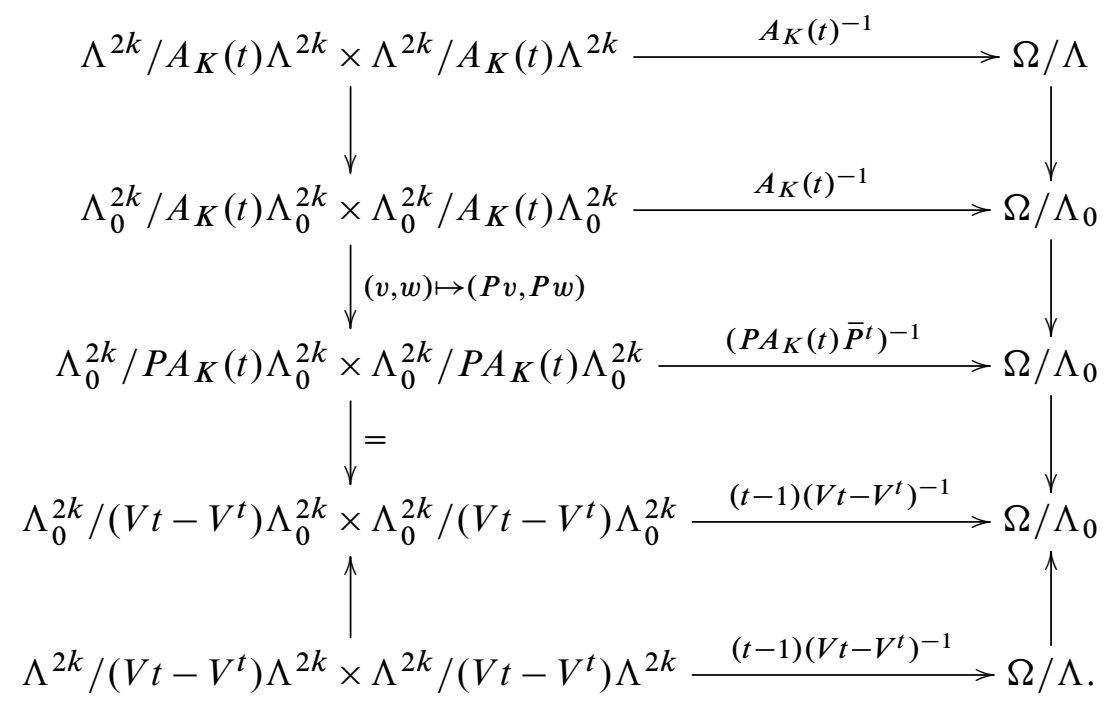

Here the top vertical maps and the bottom vertical maps are induced by the inclusion $\Lambda \rightarrow \Lambda_{0}$. Recall that multiplication by $t-1$ induces an isomorphism of $\Lambda^{2 k} /\left(V t-V^{t}\right) \Lambda^{2 k}$ and of $\Lambda^{2 k} / A_{K}(t) \Lambda^{2 k}$ (see [41]). It follows that the two aforementioned maps are isomorphisms of $\Lambda$-modules. For the third vertical map we made use of the fact that

$$
P A_{K}(t) \bar{P}^{t}=(t-1)^{-1}\left(V t-V^{t}\right)
$$

and we used that

$$
P A_{K}(t) \Lambda_{0}^{2 k}=P A_{K}(t) \bar{P}^{t} \Lambda_{0}^{2 k}=(t-1)^{-1}\left(V t-V^{t}\right) \Lambda_{0}^{2 k}=\left(V t-V^{t}\right) \Lambda_{0}^{2 k} .
$$

Since all vertical maps on the left in the above commutative diagram are isomorphisms we deduce from (2-5) that $\lambda\left(A_{K}(t)\right) \cong \lambda(K)$.

We conclude this section with the following lemma:

Lemma 2.2 Let $V$ be a matrix which is $S$-equivalent to a Seifert matrix of the knot $K$ and such that $V$ satisfies (2-2). Let $A_{K}(t)$ be the matrix as in (2-3). Then the matrix $A(t)=A_{K}(t) \oplus(1)$ (ie the block diagonal sum of the matrices $A_{K}(t)$ and (1)) represents $\lambda(K)$ and the bilinear matrix $A(1)$ is diagonalizable over $\mathbb{Z}$.

Proof Let $V$ be a Seifert matrix of the knot $K$ of size $2 k$ satisfying (2-2). Then we can write

$$
V=\left(\begin{array}{cc}
B & C+I \\
C^{t} & D
\end{array}\right)
$$


where $B, C$ and $D$ are $k \times k$ matrices, $I$ is the identity matrix and moreover $B=B^{t}$, $D=D^{t}$. It is easy to compute that

$$
A_{K}(1)=\left(\begin{array}{cc}
B & -I \\
-I & 0
\end{array}\right) \text {. }
$$

It is straightforward to verify that $A_{K}(1)$ is congruent over $\mathbb{Q}$ to the block sum of $I$ and $-I$, hence $A_{K}(1)$, viewed as a symmetric bilinear pairing, is indefinite. If we consider $A(t)=A_{K}(t) \oplus$ (1) (which clearly represents the same Blanchfield pairing as $A_{K}(t)$ ), then $A(1)$ is an indefinite, odd symmetric bilinear pairing over $\mathbb{Z}$, hence by Husemoller and Milnor [22, Theorem 4.3] it is diagonalizable.

\subsection{Definition of $n(K)$}

Let $K \subset S^{3}$ be a knot. It follows from Lemma 2.2 that it makes sense to define $n(K)$ as the minimal size of a hermitian matrix $A$ over $\mathbb{Z}\left[t^{ \pm 1}\right]$ such that

- $\lambda(A) \cong \lambda(K)$

- the matrix $A(1)$ is congruent over $\mathbb{Z}$ to a diagonal matrix which has \pm 1 on the diagonal.

In fact we can use Lemma 2.2 to deduce a more precise statement.

Lemma 2.3 For any knot $K$ we have the inequality

$$
n(K) \leq \operatorname{deg} \Delta_{K}(t)+1 .
$$

Proof It is well known (see eg Levine [40, page 195]) that any Seifert matrix is $S$-equivalent to a matrix $V$ which is nonsingular and which satisfies (2-2). Let $2 k$ be the size of $V$. Then we have that $t^{-k} \operatorname{det}\left(V t-V^{t}\right)=\Delta_{K}(t)$, and it follows easily that $V$ is a matrix of size $\operatorname{deg} \Delta_{K}(t)$. The corollary now follows immediately from Lemma 2.2.

Remark (1) Suppose that

$$
V=\left(\begin{array}{cc}
B & C+I \\
C^{t} & D
\end{array}\right)
$$

is a matrix of size $\operatorname{deg} \Delta_{K}(t)$ which is $S$-equivalent to a Seifert matrix of $K$ and $B=B^{t}, D=D^{t}$. If $B$ itself represents an odd pairing, then $A_{K}(1)$ is already diagonalizable. In that case $n(K) \leq \operatorname{deg} \Delta_{K}(t)$. 
(2) Fogel [13, Section 3.3] gives examples of two knots $K_{1}$ and $K_{2}$ such that $n\left(K_{1} \# K_{2}\right)=n\left(K_{1}\right)=n\left(K_{2}\right)=1$. This shows that the $n(K)$ invariant is in general not additive. This is in contrast to the conjecture that the unknotting number is additive (see Kirby [33, Problem 1.69(B)] and Scharlemann [62] for some strong evidence towards this conjecture).

\subsection{The Blanchfield pairing and intersection pairings on 4-manifolds}

We now turn to the proof that $u_{a}(K) \geq n(K)$. We will show that the 0 -framed surgery on a knot which can be turned into an Alexander polynomial one knot using $u_{+}$positive and $u_{-}$negative crossing changes cobounds a 4-manifold with certain properties. We will then show that a matrix representing the equivariant intersection pairing on that 4-manifold gives in fact a presentation matrix for the Blanchfield pairing of $K$.

Given a knot $K \subset S^{3}$ we denote in the following by $M(K)$ the 0 -framed surgery on $K$. Furthermore, given a topological 4 -manifold $W$ with boundary $M$, we consider the sequence of maps

$$
H_{2}(W ; \mathbb{Z}) \stackrel{\iota}{\rightarrow} H_{2}(W, M ; \mathbb{Z}) \stackrel{\mathrm{PD}}{\longrightarrow} H^{2}(W ; \mathbb{Z}) \stackrel{\text { ev }}{\rightarrow} \operatorname{Hom}_{\mathbb{Z}}\left(H_{2}(W ; \mathbb{Z})\right),
$$

where $\iota$ denotes the inclusion induced map, PD denotes Poincaré duality and ev denotes the evaluation map. This defines a pairing

$$
H_{2}(W ; \mathbb{Z}) \times H_{2}(W ; \mathbb{Z}) \rightarrow \mathbb{Z},
$$

called the ordinary intersection pairing of $W$, which is well known to be symmetric. In the following we will several times make implicit use of the following lemma.

Lemma 2.4 Suppose the following hold:

(1) $M$ is connected.

(2) $\quad H_{1}(M ; \mathbb{Z}) \rightarrow H_{1}(W ; \mathbb{Z})$ is an isomorphism.

(3) $H_{1}(W ; \mathbb{Z})$ is torsion free.

Then the ordinary intersection pairing is nonsingular.

Proof The assumption that $H_{1}(M ; \mathbb{Z}) \rightarrow H_{1}(W ; \mathbb{Z})$ is an isomorphism implies by Poincaré duality that $H^{2}(M ; \mathbb{Z}) \rightarrow H^{3}(W, M ; \mathbb{Z})$ is an isomorphism. From the universal coefficient theorem, $\operatorname{Hom}_{\mathbb{Z}}\left(H_{2}(M ; \mathbb{Z}), \mathbb{Z}\right) \rightarrow \operatorname{Hom}_{\mathbb{Z}}\left(H_{3}(W, M ; \mathbb{Z}), \mathbb{Z}\right)$ is an isomorphism. But $H_{2}(M ; \mathbb{Z}) \cong H^{1}(M ; \mathbb{Z})$ and $H_{3}(W, M ; \mathbb{Z}) \cong H^{1}(W ; \mathbb{Z}) \cong$ $\operatorname{Hom}\left(H_{1}(W ; \mathbb{Z}), \mathbb{Z}\right)$ are torsion free, it thus follows that $H_{3}(W, M ; \mathbb{Z}) \rightarrow H_{2}(M ; \mathbb{Z})$ 
is an isomorphism. It follows from the long exact sequence of the pair $(W, M)$ that the map $\iota: H_{2}(W ; \mathbb{Z}) \rightarrow H_{2}(W, M ; \mathbb{Z})$ is an isomorphism. The assumption that $H_{1}(W ; \mathbb{Z})$ is torsion free implies by the universal coefficient theorem that the evaluation map ev: $H^{2}(W ; \mathbb{Z}) \rightarrow \operatorname{Hom}_{\mathbb{Z}}\left(H_{2}(W ; \mathbb{Z}), \mathbb{Z}\right)$ is an isomorphism. It now follows that the ordinary intersection pairing is nonsingular.

We now consider a topological 4 -manifold $W$ with boundary $M$ such that $\pi_{1}(W) \cong \mathbb{Z}$. We then consider the sequence of maps

$$
H_{2}(W ; \Lambda) \stackrel{\iota}{\rightarrow} H_{2}(W, M ; \Lambda) \stackrel{\mathrm{PD}}{\longrightarrow} \overline{H^{2}(W ; \Lambda)} \stackrel{\text { ev }}{\rightarrow} \overline{\operatorname{Hom}_{\Lambda}\left(H_{2}(W ; \Lambda), \Lambda\right)},
$$

where the first map is again the inclusion induced map, the second map is Poincaré duality and the third map is the evaluation map. This composition of maps defines a pairing

$$
H_{2}(W ; \Lambda) \times H_{2}(W ; \Lambda) \rightarrow \Lambda,
$$

which is well known to be hermitian. We refer to this pairing as the twisted intersection pairing on $W$. Now we introduce the following notion, which we shall use several times in the future.

Definition 2.5 Let $K$ be a knot and $M(K)$ the zero framed surgery on $K$. We shall say that a 4-manifold $W$ tamely cobounds $M(K)$ if the following conditions are satisfied:

(1) $\partial W=M(K)$.

(2) The inclusion induced map $H_{1}(M(K) ; \mathbb{Z}) \rightarrow H_{1}(W ; \mathbb{Z})$ is an isomorphism.

(3) $\pi_{1}(W) \cong \mathbb{Z}$.

If furthermore the intersection form on $H_{2}(W ; \mathbb{Z})$ is diagonalizable, we say that $W$ strictly cobounds $M(K)$.

The following theorem will be the key ingredient in the proof that $u_{a}(K) \geq n(K)$.

Theorem 2.6 Let $K$ be a knot. Suppose there exists a topological 4-manifold $W$, which tamely cobounds $M(K)$. Then $H_{2}(W ; \Lambda)$ is free of rank $b_{2}(W)$. Furthermore, if $B$ is an integral matrix representing the ordinary intersection pairing of $W$, then there exists a basis $\mathcal{B}$ for $H_{2}(W ; \Lambda)$ such that the matrix $A(t)$ representing the twisted intersection pairing with respect to $\mathcal{B}$ has the following two properties:

(1) $\lambda(A(t)) \cong \lambda(K)$,

(2) $A(1)=B$.

The proof of Theorem 2.6 is rather long and will require all of the following section. 


\subsection{Proof of Theorem 2.6}

Let $K$ be a knot and let $W$ be a topological 4-manifold $W$, which tamely cobounds $M(K)$. Throughout this section we write $M:=M(K)$. We first want to prove the following lemma:

Lemma 2.7 The $\Lambda$-module $H_{2}(W ; \Lambda)$ is free of rank $b_{2}(W)$.

Proof We first want to show that $H_{2}(W ; \Lambda)$ is a free $\Lambda$-module. Note that $H_{2}(W ; \Lambda)$ is a finitely generated $\Lambda$-module since $\Lambda$ is Noetherian. By Kawauchi [25, Corollary 3.7] the module $H_{2}(W ; \Lambda)$ is free if and only if $\operatorname{Ext}_{\Lambda}^{i}\left(H_{2}(W ; \Lambda), \Lambda\right)=0$ for $i=1,2$.

Note that $\pi_{1}(W) \cong \mathbb{Z}$ implies that $H_{1}(W ; \Lambda)=0$. We also have $H_{4}(W ; \Lambda)=0$. We furthermore have an isomorphism $H_{0}(M ; \Lambda) \rightarrow H_{0}(W ; \Lambda)$. We thus conclude from the long exact homology sequence corresponding to the pair $(W, M)$ that $H_{0}(W, M ; \Lambda)=0$ and $H_{1}(W, M ; \Lambda)=0$.

Recall that the UCSS (see Section 2.1) starts with $E_{2}^{p, q}=\operatorname{Ext}_{\Lambda}^{q}\left(H_{p}(W ; \Lambda), \Lambda\right)$ and converges to $H^{*}(W ; \Lambda)$. Furthermore the differentials have degree $(1-r, r)$. By the above we have $E_{2}^{p, q}=0$ for $p=1$ and $p=4$. Since $\Lambda$ has global dimension 2 we also have $E_{2}^{p, q}=0$ for $q \geq 3$. Finally note that

$$
E_{2}^{0,2}=\operatorname{Ext}_{\Lambda}^{2}\left(H_{0}(W ; \Lambda), \Lambda\right)=\operatorname{Ext}_{\Lambda}^{2}(\Lambda /(t-1) \Lambda, \Lambda)=0 .
$$

It now follows from the UCSS that we have a monomorphism

$$
E_{2}^{2,1}=\operatorname{Ext}_{\Lambda}^{1}\left(H_{2}(W ; \Lambda), \Lambda\right) \rightarrow H^{3}(W ; \Lambda) .
$$

But $H^{3}(W ; \Lambda) \cong \overline{H_{1}(W, M ; \Lambda)}=0$. Similarly, it follows from the UCSS that we have a monomorphism

$$
E_{2}^{2,2}=\operatorname{Ext}_{\Lambda}^{2}\left(H_{2}(W ; \Lambda), \Lambda\right) \rightarrow H^{4}(W ; \Lambda) .
$$

But $H^{4}(W ; \Lambda) \cong \overline{H_{0}(W, M ; \Lambda)}=0$. This concludes the proof of the claim that $\mathrm{H}_{2}(W ; \Lambda)$ is a free module.

We now turn to the proof that $H_{2}(W ; \Lambda)$ is a free $\Lambda$-module of rank $s:=b_{2}(W)$. It remains to show that $H_{2}(W ; \Lambda)$ is of rank $s$. Since $\Omega$ is flat over $\Lambda$, it suffices to show that $\operatorname{dim}_{\Omega}\left(H_{2}(W ; \Omega)\right)=s$. It is clear that $H_{i}(W ; \Omega)=0$ for $i=0,1,4$. Furthermore $H_{3}(W ; \Omega) \cong \overline{H^{1}(W, M ; \Omega)}$. But since $\Omega$ is a field the latter is isomorphic to $\overline{H_{1}(W, M ; \Omega)}$ which is zero. We thus calculate

$$
\operatorname{dim}_{\Omega}\left(H_{2}(W ; \Omega)\right)=\sum_{i=0}^{4}(-1)^{i} \operatorname{dim}_{\Omega}\left(H_{i}(W ; \Omega)\right)=\chi(W) .
$$


Now note that $b_{0}(W)=b_{1}(W)=1$ and $b_{4}(W)=0$. Also note that $H^{3}(W ; \mathbb{Z}) \cong$ $H_{1}(W, M ; \mathbb{Z})=0$ since we assume that $H_{1}(M ; \mathbb{Z}) \rightarrow H_{1}(W ; \mathbb{Z})$ is an isomorphism. It thus follows that $b_{3}(W)=0$, and we see that $\chi(W)=b_{2}(W)=s$. This concludes the proof of the lemma.

We now write $s=b_{2}(W)$. We pick a basis $\mathcal{B}$ for $H_{2}(W ; \Lambda)$ and denote by $A=A(t)$ the corresponding $s \times s$-matrix representing the twisted intersection pairing. Note that $A$ is a hermitian $s \times s$-matrix. By the argument of Friedl, Hambleton, Melvin and Teichner [16, Lemma 2.2] we see that the matrix $A(1)$ represents the ordinary intersection pairing on $\mathrm{H}_{2}(W ; \mathbb{Z})$. In particular there exists an integral matrix $P$ such that $P A(1) P^{t}=B$. After acting on the basis $\mathcal{B}$ by the matrix $P$ we can without loss of generality assume that in fact $A(1)=B$. The following lemma now concludes the proof of Theorem 2.6.

Lemma 2.8 The pairing $\lambda(A)$ is isometric to $\lambda(K)$.

The proof of the lemma will require the remainder of this section. We first want to prove the following claim:

Claim The following is a short exact sequence:

$$
0 \rightarrow H_{2}(W ; \Lambda) \rightarrow H_{2}(W, M ; \Lambda) \rightarrow H_{1}(M ; \Lambda) \rightarrow 0
$$

Proof To prove the claim we first consider the following exact sequence:

$$
H_{2}(M ; \Lambda) \rightarrow H_{2}(W ; \Lambda) \rightarrow H_{2}(W, M ; \Lambda) \rightarrow H_{1}(M ; \Lambda) \rightarrow H_{1}(W ; \Lambda) \rightarrow \cdots
$$

Recall that $H_{1}(W ; \Lambda)=0$. Also note that

$$
H_{2}(M ; \Lambda) \otimes_{\Lambda} \Omega \cong H_{2}(M ; \Omega) \cong \overline{H^{1}(M ; \Omega)} \cong \overline{\operatorname{Hom}_{\Omega}\left(H_{1}(M ; \Omega), \Omega\right)}=0,
$$

since $H_{1}(M ; \Omega)=H_{1}(M ; \Lambda) \otimes_{\Lambda} \Omega=0$ (here we used that $H_{1}(M ; \Lambda)$ is torsion). In particular $H_{2}(M ; \Lambda)$ is torsion and the map $H_{2}(M ; \Lambda) \rightarrow H_{2}(W ; \Lambda)$ is trivial since $H_{2}(W ; \Lambda)$ is a free $\Lambda$-module. This now concludes the proof of the claim.

We now define a Blanchfield pairing on $H_{1}(M ; \Lambda)$ and an intersection pairing on $\mathrm{H}_{2}(W, M ; \Lambda)$. First of all, similar to $(2-1)$ we can consider the following sequence of isomorphisms:

$$
H_{1}(M ; \Lambda) \stackrel{\mathrm{PD}}{\longrightarrow} \overline{H^{2}(M ; \Lambda)} \cong \overline{H^{1}(M ; \Omega / \Lambda)} \stackrel{\mathrm{ev}}{\rightarrow} \operatorname{Hom}_{\Lambda}\left(H_{1}(M ; \Lambda), \Omega / \Lambda\right)
$$

This defines a hermitian nonsingular pairing

$$
H_{1}(M ; \Lambda) \times H_{1}(M ; \Lambda) \rightarrow \Omega / \Lambda .
$$


It is well known that the natural map $H_{1}(X(K) ; \Lambda) \rightarrow H_{1}(M ; \Lambda)$ is an isomorphism, and it follows immediately that the Blanchfield pairing on $X(K)$ is isometric to the pairing (2-8) on $M$.

Secondly, we consider the sequence of maps

$$
\begin{aligned}
H_{2}(W, M ; \Lambda) & \stackrel{\mathrm{PD}}{\rightarrow} \overline{H^{2}(W ; \Lambda)} \rightarrow \overline{H^{2}(W ; \Omega)} \cong \overline{H^{2}(W, M ; \Omega)} \\
& \stackrel{\text { ev }}{\rightarrow} \overline{\operatorname{Hom}_{\Lambda}\left(H_{2}(W, M ; \Lambda), \Omega\right) .}
\end{aligned}
$$

Here, for the third map we made use of the fact that $H_{1}(M ; \Lambda)$ is $\Lambda$-torsion, therefore (2-7) implies that the inclusion induced map $H^{2}(W, M ; \Omega) \rightarrow H^{2}(W ; \Omega)$ is an isomorphism. The other maps in (2-9) are given by Poincaré duality, inclusion of rings and the evaluation homomorphism. The sequence of maps in (2-9) now defines a hermitian pairing

$$
H_{2}(W, M ; \Lambda) \times H_{2}(W, M ; \Lambda) \rightarrow \Omega \text {. }
$$

Claim The intersection pairing on $W$, the intersection pairing on $H_{2}(W, M ; \Lambda)$ and the Blanchfield pairing on $M$ fit into the following commutative diagram, where the left vertical maps form a short exact sequence:

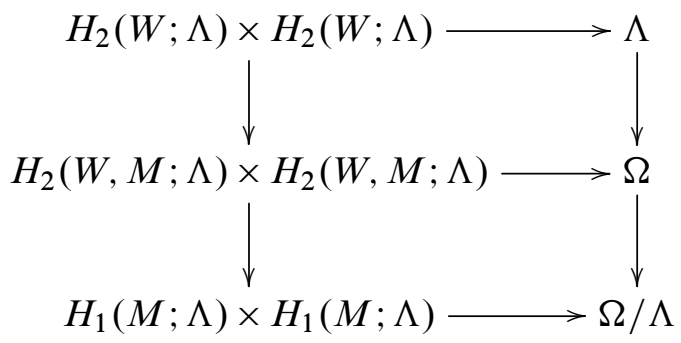

Proof In the previous claim we already showed that the left vertical maps form a short exact sequence. We now consider the following diagram:

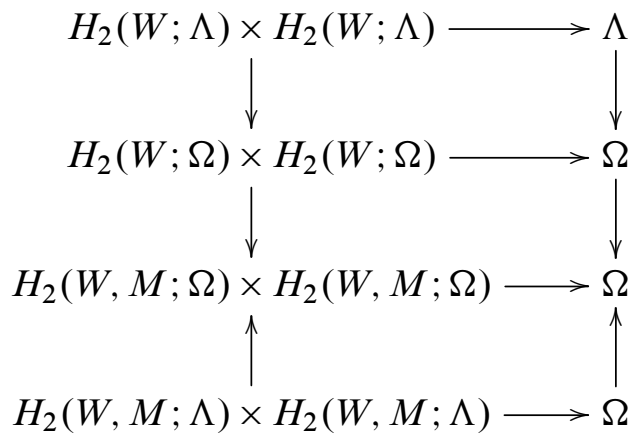


The pairings on $\Omega$-homology are defined in complete analogy to the corresponding pairings on $\Lambda$-homology, and the vertical maps are the obvious maps. It now follows easily from the definitions that this is a commutative diagram. Since the image of $H_{2}(W ; \Lambda) \rightarrow H_{2}(W, M ; \Omega)$ lies in the image of $H_{2}(W, M ; \Lambda) \rightarrow H_{2}(W, M ; \Omega)$ it now follows that the top square in the diagram of the claim commutes.

We now consider the following diagram:

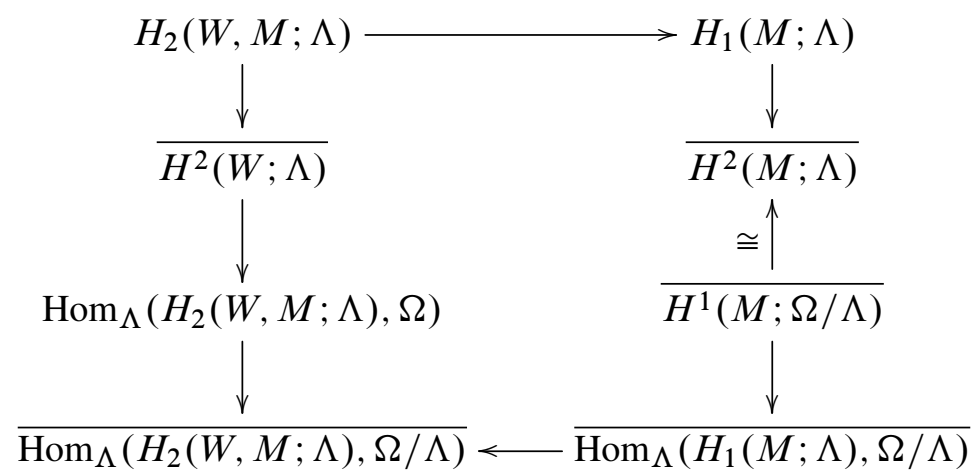

where the left middle vertical map is a part of the definition of the intersection pairing on $\mathrm{H}_{2}(W, M ; \Lambda)$. Furthermore the horizontal maps are the maps induced by long exact sequences corresponding to the pair $(W, M)$. By Leidy [38, Section 6] this diagram commutes. This now implies that the lower square in the claim also commutes.

Claim The evaluation map

$$
H^{2}(W ; \Lambda) \stackrel{\text { ev }}{\rightarrow} \operatorname{Hom}_{\Lambda}\left(H_{2}(W ; \Lambda), \Lambda\right)
$$

is an isomorphism.

Proof In order to prove the claim we have to study the UCSS corresponding to $H^{2}(W ; \Lambda)$. Note that $\operatorname{Ext}_{\Lambda}^{1}\left(H_{0}(W ; \Lambda), \Lambda\right)=\Lambda /(t-1) \Lambda$ is $\Lambda$-torsion, hence the differential

$$
d_{2}: E_{2}^{0,1}=\operatorname{Ext}_{\Lambda}^{1}\left(H_{0}(W ; \Lambda), \Lambda\right) \rightarrow E_{2}^{2,0}=\operatorname{Ext}_{\Lambda}^{0}\left(H_{2}(W ; \Lambda), \Lambda\right)
$$

is zero since $\operatorname{Ext}_{\Lambda}^{0}\left(H_{2}(W ; \Lambda), \Lambda\right)=\operatorname{Hom}_{\Lambda}\left(H_{2}(W ; \Lambda), \Lambda\right)$ is $\Lambda$-torsion free. It now follows (using the earlier discussion) that the UCSS for $H^{2}(W ; \Lambda)$ gives rise to the desired isomorphism:

$$
H^{2}(W ; \Lambda) \stackrel{\cong}{\rightrightarrows} \operatorname{Ext}_{\Lambda}^{0}\left(H_{2}(W ; \Lambda), \Lambda\right)=\operatorname{Hom}_{\Lambda}\left(H_{2}(W ; \Lambda), \Lambda\right) .
$$

This concludes the proof. 
Recall that we picked a basis $\mathcal{B}$ for $H_{2}(W ; \Lambda)$ and that we denote by $A=A(t)$ the corresponding matrix representing the twisted intersection pairing on $H_{2}(W ; \Lambda)$. Now note that by Poincaré duality and by the above claim we have two isomorphisms

$$
H_{2}(W, M ; \Lambda) \stackrel{\mathrm{PD}}{\cong} \overline{H^{2}(W ; \Lambda)} \stackrel{\mathrm{ev}}{\cong} \overline{\operatorname{Hom}_{\Lambda}\left(H_{2}(W ; \Lambda), \Lambda\right)} .
$$

We now endow $H_{2}(W, M ; \Lambda)$ with the basis $\mathcal{C}$ which is dual to $\mathcal{B}$. It follows easily from (2-6) and (2-13) that the inclusion induced map $H_{2}(W ; \Lambda) \rightarrow H_{2}(W, M ; \Lambda)$ with respect to the bases $\mathcal{B}$ and $\mathcal{C}$ is given by $A$.

We now rewrite the diagram (2-10) in terms of our bases, we thus obtain the following diagram:

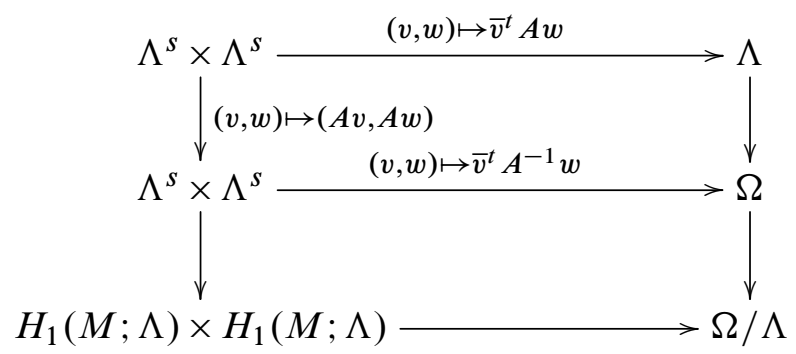

The statement of Lemma 2.8 now follows from this diagram and the fact that the left vertical maps form a short exact sequence. This concludes the proof of Theorem 2.6.

\subsection{Proof of Theorem 1.1}

Clearly the following theorem, combined with Theorem 2.6, implies Theorem 1.1 from the introduction.

Theorem 2.9 Let $K$ be a knot such that $u_{+}$positive crossing changes and $u_{-}$negative crossing changes turn $K$ into an Alexander polynomial one knot $J$. Then there exists an oriented topological 4-manifold $W$ which strictly cobounds $M(K)$. Moreover, the intersection pairing on $\mathrm{H}_{2}(W ; \mathbb{Z})$ is represented by a diagonal matrix of size $u_{+}+u_{-}$ such that $u_{+}$entries are equal to -1 and $u_{-}$entries are equal to +1 .

Proof We first recall the following well known reinterpretation of a crossing change. Let $K \subset S^{3}$ be a knot. Suppose we perform an $\epsilon$-crossing change along a crossing. We denote by $D \subset S^{3}$ an embedded disk which intersects $K$ in precisely two points with opposite orientations, one point on each strand involved in the crossing change. If we now perform $\epsilon$-surgery on the curve $c=\partial D$, then the resulting 3 -manifold $\Sigma$ is diffeomorphic to $S^{3}$, and $K \subset \Sigma$ is the result of performing an $\epsilon$-crossing change. 
In the following we will use the following notation: let $c_{1}, \ldots, c_{s}$ be simple closed curves which form the unlink in $S^{3}$ and let $\epsilon_{1}, \ldots, \epsilon_{s} \in\{-1,1\}$; then we denote by $\Sigma\left(c_{1}, \ldots, c_{s}, \epsilon_{1}, \ldots, \epsilon_{s}\right)$ the result of performing $\epsilon_{i}$-surgery along $c_{i}$ for $i=1, \ldots, s$. Note that this 3-manifold is diffeomorphic to the standard 3-sphere.

Let $K$ be a knot such that $u_{+}$positive crossing changes and $u_{-}$negative crossing changes turn $K$ into an Alexander polynomial one knot $J$. Put differently, there exists an Alexander polynomial one knot $J$ such that $u_{+}$negative crossing changes and $u_{-}$ negative positive changes turn $J$ into $K$. We write $s=u_{+}+u_{-}$and $n_{i}=-1$ for $i=1, \ldots, u_{+}$and $n_{i}=1$ for $i=u_{+}+1, \ldots, u_{+}+u_{-}$. By the above discussion there exist simple closed curves $c_{1}, \ldots, c_{s}$ in $X(J)$ with the following properties:

(1) $c_{1}, \ldots, c_{s}$ are the unlink in $S^{3}$.

(2) The linking numbers $\operatorname{lk}\left(c_{i}, J\right)$ are zero.

(3) The image of $J$ in

$$
\Sigma\left(c_{1}, \ldots, c_{s}, n_{1}, \ldots, n_{s}\right)
$$

is the knot $K$.

Note that the curves $c_{1}, \ldots, c_{s}$ lie in $S^{3} \backslash v J$ and we can thus view them as lying in $M(J)$. The manifold $M(K)$ is then the result of $n_{i}$ surgery on $c_{i} \subset M(J)$ for $i=1, \ldots, s$.

Since $J$ is a knot with trivial Alexander polynomial it follows from Freedman's theorem (see Freedman and Quinn [15, Theorem 117B]), that $J$ is topologically slice. In fact there exists a locally flat slice disk $D \subset D^{4}$ for $J$ such that $\pi_{1}\left(D^{4} \backslash D\right)=\mathbb{Z}$. We now write $X:=D^{4} \backslash v D$. Then $X$ is an oriented topological 4 -manifold $X$ with the following properties:

(1) $\partial X=M(J)$ as oriented manifolds.

(2) $\pi_{1}(X) \cong \mathbb{Z}$.

(3) $H_{1}(M(J) ; \mathbb{Z}) \rightarrow H_{1}(X ; \mathbb{Z})$ is an isomorphism.

(4) $H_{2}(X ; \mathbb{Z})=0$.

We denote by $W$ the 4-manifold which is the result of adding 2-handles along $c_{1}, \ldots, c_{s} \subset M(J)$ with framings $n_{1}, \ldots, n_{s}$ to $X$. Note that $\partial W=M(K)$ as oriented manifolds. We will henceforth write $M=M(K)$. Note that the curves $c_{1}, \ldots, c_{s}$ are nullhomologous, it follows easily that the map $H_{1}(M ; \mathbb{Z}) \rightarrow H_{1}(W ; \mathbb{Z})$ is an isomorphism and that $\pi_{1}(W) \cong \mathbb{Z}$. It thus remains to prove the following claim: 
Claim The ordinary intersection pairing on $W$ is represented by a diagonal matrix of size $u_{+}+u_{-}$such that $u_{+}$diagonal entries are equal to -1 and $u_{-}$diagonal entries are equal to 1 .

Recall that the curves $c_{1}, \ldots, c_{s}$ form the unlink in $S^{3}$ and that the linking numbers $\operatorname{lk}\left(c_{i}, J\right)$ are zero. In particular the curves $c_{1}, \ldots, c_{s}$ are also nullhomologous in $M(J)$. It is clear that we can now find disjoint surfaces $F_{1}, \ldots, F_{S}$ in $M(J) \times[0,1]$ such that $\partial F_{i}=c_{i} \times 1$. By adding the cores of the 2-handles attached to the $c_{i}$ we now obtain closed surfaces $C_{1}, \ldots, C_{s}$ in $W$. It is straightforward to see that $C_{i} \cdot C_{j}=0$ for $i \neq j$ and $C_{i} \cdot C_{i}=n_{i}$. A Mayer-Vietoris argument shows that the surfaces $C_{1}, \ldots, C_{s}$ present a basis for $H_{2}(W ; \mathbb{Z})$. In particular the intersection matrix on $W$ with respect to this basis is given by $\left(C_{i} \cdot C_{j}\right)$, ie it is a diagonal matrix such that $u_{+}$diagonal entries are equal to -1 and $u_{-}$diagonal entries are equal to 1 . This concludes the proof of the claim.

Remark In the proof of Theorem 2.9 (and thus in the proof that $u_{a}(K) \geq n(K)$ ), we made use of Freedman's theorem that a knot with trivial Alexander polynomial is topologically slice. This deep topological fact is not necessary to prove Theorem 1.1, but it simplifies the algebra and the exposition.

If a knot $K$ has unknotting number $u$, then Montesinos [47] has shown that the 2-fold branched cover $\Sigma(K)$ is given by Dehn surgery on some framed link in $S^{3}$ with $u$ components, with half-integral framing coefficients. This fact is used in the original proof of the Lickorish obstruction and it lies at the heart of some of the deepest results on unknotting numbers (see eg $[51 ; 55]$ ) which are obtained by studying Heegaard-Floer invariants of the compact 3-manifold $\Sigma(K)$.

Let $K$ be a knot such that $u_{+}$positive crossing changes and $u_{-}$negative crossing changes turn $K$ into the unknot. If we take $X=S^{1} \times D^{3}$ in the proof of Theorem 2.9, then we immediately see that there exists an oriented smooth 4 -manifold $W$ which satisfies the properties (1)-(4) of Theorem 2.9. This suggests that further information on unknotting numbers can be obtained from considering higher cyclic covers (or the infinite cyclic cover) of $M(K)$.

\section{The Blanchfield pairing and the linking pairing}

In this section we will relate the Blanchfield pairing to the linking pairing on the homology of the $2-$ fold branched cover of a given knot $K$. 


\subsection{Preliminary results}

The following proposition is a key tool in relating $n(K)$ to other invariants:

Proposition 3.1 (Ranicki [56, Proposition 1.7.1]) Let $A$ and $B$ be hermitian matrices over $\mathbb{Z}\left[t^{ \pm 1}\right]$ with $\operatorname{det}(A(1))=\operatorname{det}(B(1))= \pm 1$. Then $\lambda(A) \cong \lambda(B)$ if and only if $A$ and $B$ are related by a sequence of the following three moves:

(1) Replace $C$ by $P C \bar{P}^{t}$, where $P$ is a matrix over $\mathbb{Z}\left[t^{ \pm 1}\right]$ with $\operatorname{det}(P)= \pm 1$.

(2) Replace $C$ by the block sum $C \oplus D$, where $D$ is a hermitian matrix over $\mathbb{Z}\left[t^{ \pm 1}\right]$ with $\operatorname{det}(D)= \pm 1$.

(3) The inverse of (2).

We can now prove the following lemma:

Lemma 3.2 Let $A(t)$ be a hermitian matrix over $\mathbb{Z}\left[t^{ \pm 1}\right]$ with $\lambda(A(t)) \cong \lambda(K)$. Then

$$
\begin{aligned}
& \operatorname{sign}(A(z))-\operatorname{sign}(A(1))=\sigma_{z}(K) \quad \text { for any } z \in S^{1}, \\
& \operatorname{null}(A(z))=\eta_{z}(K) \quad \text { for any } z \in \mathbb{C} \backslash\{0,1\} \text {. }
\end{aligned}
$$

Proof First let $D(t)$ be any hermitian matrix over $\mathbb{Z}\left[t^{ \pm 1}\right]$. It is well known that the function

$$
S^{1} \rightarrow \mathbb{Z}, \quad z \mapsto \operatorname{sign}(D(z)) .
$$

is constant outside of the set of zeros of $\operatorname{det}(D(t))$. In particular if $\operatorname{det}(D(t))= \pm 1$, then the signature function is constant. It now follows easily from Proposition 2.1 that if $A(t)$ and $B(t)$ are hermitian matrices over $\mathbb{Z}\left[t^{ \pm 1}\right]$ with $\lambda(A(t)) \cong \lambda(B(t))$, then

$$
\operatorname{sign}(A(z))-\operatorname{sign}(A(1))=\operatorname{sign}(B(z))-\operatorname{sign}(B(1)) \quad \text { for any } z \in S^{1} .
$$

The first claim now follows from (2-4) and Proposition 2.1. The proof of the second statement also follows from a similar argument.

\subsection{Linking pairings}

We will now relate the Blanchfield pairing to the linking pairing on the 2-fold branched cover. Later on, this will allow us to relate $n(K)$ to the Lickorish obstruction and the Jabuka obstruction, as well as to get new computable obstructions to $n(K)=2$ or $n(K)=3$. 
A linking pairing is a nonsingular symmetric bilinear pairing $H \times H \rightarrow \mathbb{Q} / \mathbb{Z}$, where $H$ is a finite abelian group of odd order. If $l$ and $l^{\prime}$ are isometric linking pairings, then we write $l \cong l^{\prime}$. An example is the linking pairing $l(K)$ defined on $H_{1}(\Sigma(K))$.

Given a symmetric integral matrix $A$ with $\operatorname{det}(A)$ odd we denote by $l(A)$ the linking pairing which is defined as

$$
\begin{aligned}
\mathbb{Z}^{n} / A \mathbb{Z}^{n} \times \mathbb{Z}^{n} / A \mathbb{Z}^{n} & \rightarrow \mathbb{Q} / \mathbb{Z}, \\
(v, w) & \mapsto v^{t} A^{-1} w .
\end{aligned}
$$

Given a linking pairing $l: H \times H \rightarrow \mathbb{Q} / \mathbb{Z}$ and $n \in \mathbb{Z}$, coprime to $|H|$, we denote by $n \cdot l$ the linking pairing given by $(n \cdot l)(v, w):=n \cdot l(v, w)$.

We can now formulate and prove the following lemma.

Lemma 3.3 Let $K$ be a knot and let $A(t)$ be a hermitian matrix over $\mathbb{Z}\left[t^{ \pm 1}\right]$ such that $\lambda(A(t)) \cong \lambda(K)$. Then

$$
l(A(-1)) \cong 2 l(K)
$$

Proof We now denote by $\mathcal{M}$ the set of all hermitian matrices $A$ over $\mathbb{Z}\left[t^{ \pm 1}\right]$ such that $\operatorname{det}(A(1))= \pm 1$. We say that $A, B \in \mathcal{M}$ are equivalent, written $A \sim B$, if $\lambda(A)$ and $\lambda(B)$ are isometric. We furthermore denote by $\mathcal{L}$ the set of isometry classes of linking pairings. We consider the map

$$
\begin{aligned}
\Phi: \mathcal{M} & \rightarrow \mathcal{L}, \\
A(t) & \mapsto l(A(-1)) .
\end{aligned}
$$

(Note that $\operatorname{det}(A(-1)) \equiv \operatorname{det}(A(1))= \pm 1 \bmod 2$.) It follows immediately from Proposition 3.1 that the map $\Phi$ descends to a map

$$
\mathcal{M} / \sim \mathcal{L} .
$$

Let $V$ be a Seifert matrix for $K$. We define $A_{K}(t)$ as in Section 2.2. It is well known (see eg [17]) that the linking pairing $l=l(K)$ on $H_{1}(\Sigma(K) ; \mathbb{Z})$ is isometric to $l\left(V+V^{t}\right)$.

An argument analogous to the proof of Proposition 2.1 with $\Lambda$ replaced by $\mathbb{Z}, \Lambda_{0}$ replaced by $\mathbb{Z}\left[\frac{1}{2}\right]$ and $t$ replaced by -1 then shows that the linking pairing $\Phi\left(A_{K}(t)\right)$ is isometric to the pairing $2 l(K)$.

Now let $A(t)$ be a hermitian matrix over $\mathbb{Z}\left[t^{ \pm 1}\right]$ such that $\lambda(A(t)) \cong \lambda(K)$. Then $A(t) \sim A_{K}(t)$ and it follows from the above that

$$
l(A(-1))=\Phi(A(t)) \cong \Phi\left(A_{K}(t)\right) \cong 2 l(K) .
$$




\subsection{The linking pairing and the algebraic unknotting number}

In the following we refer to a positive-definite matrix as $(+1)$-definite and we refer to a negative definite matrix as a $(-1)$-definite matrix. We now have the following:

Theorem 3.4 If $n(K)=n$, then there exists a symmetric $n \times n$-matrix $A$ over $\mathbb{Z}$ which has the following three properties:

(1) $|\operatorname{det}(A)|=|\operatorname{det}(K)|$.

(2) $l(A) \cong 2 l(K)$.

(3) A modulo two equals the identity matrix.

If $\sigma(K)=2 n \cdot \epsilon$ with $\epsilon \in\{-1,1\}$, then we can furthermore arrange that $A$ has the following two properties:

(4) $A$ is $\epsilon$-definite.

(5) The diagonal entries of $A$ modulo four are equal to $-\epsilon$.

Remark Theorem 3.4 is closely related to [51, Theorem 3], which is the main technical theorem of [51]. More precisely, Owens shows in [51, Theorem 3] that if $u(K)=u$, then $\Sigma(K)$ can be obtained by Dehn surgery along a $u$-component link with a certain framing matrix. It then follows from that surgery description of $\Sigma(K)$ that there exists a $u \times u$-matrix $A$ over $\mathbb{Z}$ which has properties described in Theorem 3.4.

Proof Since $n(K)=n$ we can find a hermitian $n \times n$-matrix $B(t)$ over $\mathbb{Z}\left[t^{ \pm 1}\right]$ such that $\lambda(B) \cong \lambda(K)$ and such that $B(1)$ is diagonalizable over $\mathbb{Z}$. Note that $B(t)$ in particular represents the Alexander module, it follows that $\operatorname{det}(B(t))= \pm \Delta_{K}(t)$, ie $\operatorname{det}(B(1))= \pm 1$ and $\operatorname{det}(B(-1))= \pm \operatorname{det}(K)$.

Let $P$ be an invertible integral matrix such that $P B(1) P^{t}$ is diagonal. After replacing $B$ by $P B P^{t}$ we can thus arrange that $B(1)$ is already diagonal. We denote the diagonal entries by $\epsilon_{1}, \ldots, \epsilon_{n}$. We furthermore denote by $b_{i j}=b_{i j}(t)$ the entries of $B=B(t)$. The fact that $b_{i i}(1)=\epsilon_{i}$ and the fact that $b_{i i}\left(t^{-1}\right)=b_{i i}(t)$ implies that

$$
b_{i i}=\epsilon_{i}+(t-1)\left(t^{-1}-1\right) c_{i i}
$$

for some polynomial $c_{i i} \in \mathbb{Z}\left[t^{ \pm 1}\right]$ with $c_{i i}\left(t^{-1}\right)=c_{i i}(t)$. Furthermore, given $i \neq j$, the fact that $b_{i j}(1)=0$ implies that

$$
b_{i j}=(t-1) \cdot c_{i j}
$$


for some $c_{i j} \in \mathbb{Z}\left[t^{ \pm 1}\right]$. By Lemma 3.3 the matrix $A:=B(-1)$ represents $2 l(K)$. By the above we have $\operatorname{det}(A)= \pm \operatorname{det}(K)$. It follows immediately from the above that $A=B(-1)$ agrees with the identity matrix modulo two.

We now assume that $\sigma(K)=2 n \cdot \epsilon$ with $\epsilon \in\{-1,1\}$. It follows from Lemma 3.2 that $\operatorname{sign}(B(-1) \oplus-B(1))=\operatorname{sign}(B(-1))-\operatorname{sign}(B(1))=2 n \cdot \epsilon$. Since $B$ is an $n \times n-$ matrix this implies that $\operatorname{sign}(B(-1))=n \cdot \epsilon$ and $\operatorname{sign}(B(1))=-n \cdot \epsilon$. In particular $A=B(-1)$ is $\epsilon$-definite. Since $B(1)$ is $\epsilon$-definite it follows also that $\epsilon_{i}=-\epsilon$ for $i=1, \ldots, n$. Since $b_{i i}=\epsilon+(t-1)\left(t^{-1}-1\right) c_{i i}$ it now follows that $A$ has the desired fourth property.

\section{Comparison of classical invariants}

In this section we show $n(K)$ subsumes the classical invariants stated in Theorem 1.2. We discuss each of the criteria of Theorem 1.2 in a separate subsection.

\subsection{Lower bounds on $u_{a}(K)$ : The Nakanishi index}

Let $K$ be a knot. The first lower bounds on the unknotting number $u(K)$ were given by Wendt [71] who showed that

$$
u(K) \geq \text { minimal number of generators of } H_{1}(\Sigma(K) ; \mathbb{Z}) .
$$

(See also Kinoshita [31; 32] and [3, Section E] for further details.) These lower bounds are subsumed by the Nakanishi index. More precisely, by [50, Theorem 3] (see also Kawauchi [26, Theorem 11.5.1]) we have the inequality

$$
u(K) \geq m(K) .
$$

It is clear that if a hermitian matrix $A$ over $\mathbb{Z}\left[t^{ \pm 1}\right]$ satisfies $\lambda(K) \cong \lambda(A)$, then $A$ is also presentation matrix for the Alexander module. We thus see that

$$
n(K) \geq m(K) .
$$

Together with Theorem 1.1 this implies that $m(K)$ gives in fact a lower bound on the algebraic unknotting number. This can also be deduced from modifying the proof provided by Nakanishi.

\subsection{Lower bounds on $u_{a}(K)$ : The Levine-Tristram signatures and the nullities}

Levine-Tristram signatures are well known to give lower bounds on the topological 4genus $g_{4}^{\text {top }}(K)$ of a knot, and hence lower bounds to the algebraic unknotting number. 
(See [49], Levine [39], Tristram [68] and Taylor [65] for details.) But in fact the following stronger inequality holds:

Theorem 4.1 Let $K$ be a knot which can be turned into an Alexander polynomial one knot using $u_{+}$positive crossing changes and $u_{-}$negative crossing changes. Then for any $z \in S^{1}$ we have

$$
-2 u_{-} \leq \eta_{z}(K)+\sigma_{z}(K) \leq 2 u_{+}
$$

in particular we have

$$
n(K) \geq \mu(K):=\frac{1}{2}\left(\max \left\{\eta_{z}(K)+\sigma_{z}(K) \mid z \in S^{1}\right\}+\max \left\{\eta_{z}(K)-\sigma_{z}(K) \mid z \in S^{1}\right\}\right) .
$$

We expect that this theorem is known to the experts, but we are not aware of a proof in the literature.

Proof By Theorem 1.1 there exists a hermitian matrix $A(t)$ of size $u_{+}+u_{-}$over $\mathbb{Z}\left[t^{ \pm 1}\right]$ with the following two properties:

(1) $A(1)$ is a diagonal matrix such that $u_{+}$diagonal entries are equal to -1 and $u_{-}$ diagonal entries are equal to 1 .

(2) $\lambda(A(t)) \cong \lambda(K)$.

Now let $z \in S^{1}$. We denote by $b_{+}$(respectively $b_{-}, b_{0}$ ) the number of positive (respectively negative, zero) eigenvalues of $A(z)$. Then it follows from Lemma 3.2 that

$$
\begin{aligned}
\eta_{z}(K)+\sigma_{z}(K) & =\operatorname{null}(A(z))+\operatorname{sign}(A(z))-\operatorname{sign}(A(1)) \\
& =b_{0}+\left(b_{+}-b_{-}\right)-\left(-u_{+}+u_{-}\right) \\
& =b_{0}+b_{+}+b_{-}-\left(-u_{+}+u_{-}\right)-2 b_{-} \\
& \leq\left(u_{+}+u_{-}\right)-\left(-u_{+}+u_{-}\right)=2 u_{+} .
\end{aligned}
$$

Similarly one shows that $\eta_{z}(K)+\sigma_{z}(K) \geq-2 u_{-}$.

\subsection{Lower bounds on $u_{a}(K)$ : The Livingston invariant}

We first recall the definition of Livingston's invariant. Let $S$ be a subring of $\mathbb{Q}(t)$. We denote by $Q$ its quotient field and we denote by $W(S \rightarrow Q)$ the Witt group of nondegenerate hermitian pairings over free $S$-modules which become nonsingular after tensoring with $Q$. Put differently, $W(S \rightarrow Q)$ is the Witt group of hermitian matrices over $S$ such that the determinant is a unit in $Q$. We refer to $[22 ; 56]$ for details. 
Let $K \subset S^{3}$ be a knot. We define $\rho_{S}(K)$ to be the minimal size of a square matrix representing $A_{K}(t)$ in $W(S \rightarrow Q)$. It follows from (2-4) that for $S=\mathbb{Q}(t)$ we obtain Livingston's invariant $\rho(K)$ (see [44]). It follows immediately from the definitions that

$$
\rho(K)=\rho_{\mathbb{Q}(t)}(K) \leq \rho_{\mathbb{Z}\left[t^{ \pm 1}\right]}(K)
$$

for any knot $K$. For the reader's convenience we will provide a proof to the following proposition, which is well known to the experts:

Proposition 4.2 Let $K$ be a knot. Then

$$
2 g_{4}^{\text {top }}(K) \geq \rho_{\mathbb{Z}\left[t^{ \pm 1}\right]}(K) .
$$

We expect that $\rho_{\mathbb{Z}\left[t^{ \pm 1}\right]}(K)$ is the best possible lower bound on the topological 4-genus which can be obtained from the Seifert matrix.

Proof Let $F$ be a Seifert surface of genus $k$ for $K$. Denote by $g$ the topological 4-genus of $K$. In that case the argument provided in the appendix of [44] shows that there exist $k-g$ linearly independent curves on $F$ on which the Seifert pairing vanishes. Since the intersection pairing on $F$ is determined by the Seifert pairing it follows that the pairwise intersection numbers of the curves are zero. In particular we can extend this set of linearly independent curves to a symplectic basis on $H_{1}(F ; \mathbb{Z})$. The corresponding Seifert matrix $V$ now has the two properties

$$
V=\left(\begin{array}{ccc}
0_{k-g \times k-g} & *_{k-g \times k-g} & *_{k-g \times 2 g} \\
*_{k-g \times k-g} & *_{k-g \times k-g} & *_{k-g \times 2 g} \\
*_{2 g \times k-g} & *_{2 g \times k-g} & *_{2 g \times 2 g}
\end{array}\right) \quad \text { and } \quad V-V^{t}=\left(\begin{array}{cc}
0 & \mathrm{id}_{k} \\
-\mathrm{id}_{k} & 0
\end{array}\right),
$$

where the subscripts indicate the size of the matrix. It now follows that $A_{K}(t)$ (as defined in Section 2.2) is of the form

$$
A_{K}(t)=\left(\begin{array}{ccc}
0_{k-g \times k-g} & *_{k-g \times k-g} & *_{k-g \times 2 g} \\
*_{k-g \times k-g} & *_{k-g \times k-g} & *_{k-g \times 2 g} \\
*_{2 g \times k-g} & *_{2 g \times k-g} & *_{2 g \times 2 g}
\end{array}\right) .
$$

It is well known that one can find an invertible matrix $P$ over $\mathbb{Z}\left[t^{ \pm 1}\right]$ such that

$$
P A_{K}(t) P^{t}=\left(\begin{array}{ccc}
0_{k-g \times k-g} & B_{k-g \times k-g} & 0_{k-g \times 2 g} \\
B_{k-g \times k-g}^{t} & 0_{k-g \times k-g} & 0_{k-g \times 2 g} \\
0_{2 g \times k-g} & 0_{2 g \times k-g} & C_{2 g \times 2 g}
\end{array}\right),
$$

where $B$ is a $(k-g) \times(k-g)$ matrix and $C$ is a $2 g \times 2 g$-matrix. Then $A_{K}(t)$ and the $2 g \times 2 g$-matrix $C$ represent the same element in $W\left(\mathbb{Z}\left[t^{ \pm 1}\right] \rightarrow \mathbb{Q}(t)\right)$. 
The classical invariant $\frac{1}{2} \rho_{\mathbb{Z}\left[t^{ \pm 1}\right]}(K)$ gives a lower bound on the topological 4-ball genus and thus on the algebraic unknotting number. The following lemma now says that, as a lower bound on $u_{a}(K)$, the invariant $\frac{1}{2} \rho_{\mathbb{Z}\left[t^{ \pm 1}\right]}(K)$ is subsumed by $n(K)$.

Lemma 4.3 For any knot $K$ we have

$$
n(K) \geq \frac{1}{2} \rho_{\mathbb{Z}\left[t^{ \pm 1}\right]}(K) .
$$

Proof Recall that we denote by $\mathcal{M}$ the set of all hermitian matrices $A$ over $\mathbb{Z}\left[t^{ \pm 1}\right]$ such that $\operatorname{det}(A(1))= \pm 1$ and we write $A \sim B$ if $\lambda(A)$ and $\lambda(B)$ are isometric. We now consider the map

$$
\begin{aligned}
\mathcal{M} & \rightarrow W\left(\mathbb{Z}\left[t^{ \pm 1}\right] \rightarrow \mathbb{Q}(t)\right), \\
A(t) & \mapsto A(t) \oplus-A(1) .
\end{aligned}
$$

Note that it is well known that given a hermitian matrix $D(t)$ over $\mathbb{Z}\left[t^{ \pm 1}\right]$ with $\operatorname{det}(D(t))= \pm 1$, the pairings $D(t)$ and $D(1)$ define the same element in $W\left(\mathbb{Z}\left[t^{ \pm 1}\right] \rightarrow\right.$ $\mathbb{Q}(t)$ ) (see [56] for details). It now follows from Proposition 3.1 that the above map descends to a map

$$
\mathcal{M} / \sim W\left(\mathbb{Z}\left[t^{ \pm 1}\right] \rightarrow \mathbb{Q}(t)\right) .
$$

The lemma now follows immediately from the definitions.

Remark In [44] Livingston shows that $\rho(K)$ is completely determined by the LevineTristram signatures. In an interesting twist Livingston [44, Section 3.1] gives an example which shows that in general $\rho(K) \geq \mu(K)$. The invariant $\mu(K)$ is thus not the optimal lower bound on the algebraic unknotting number which can be obtained from the Levine-Tristram signatures and the nullities.

\subsection{The unknotting number one obstruction by Fogel, Murakami and Rickard}

The following unknotting number one obstruction was proved by Murakami [48] and Fogel [13, page 32] and it was already known to John Rickard, as we were informed by Lickorish.

Theorem 4.4 Let $K$ be a knot and let $\epsilon \in\{-1,1\}$. If $K$ can be turned into an Alexander polynomial one knot using one $\epsilon$-crossing change, then there exists a generator $g$ of $H_{1}\left(X(K) ; \mathbb{Z}\left[t^{ \pm 1}\right]\right)$ such that

$$
\lambda(g, g)=\frac{-\epsilon}{\Delta_{K}(t)} \in \mathbb{Q}(t) / \mathbb{Z}\left[t^{ \pm 1}\right] .
$$

We will now see that it is an almost immediate corollary to Theorem 1.1. 
Proof It follows from Theorem 1.1 that $\lambda(K) \cong \lambda(p(t))$ for a polynomial $p(t)$ with $p(1)=-\epsilon$. Since $p(t)$ represents the Alexander module and since $\Delta_{K}(1)=1$ it follows that $p(t)=-\epsilon \Delta_{K}(t)$. In particular there exists a generator $g$ of $H_{1}\left(X(K) ; \mathbb{Z}\left[t^{ \pm 1}\right]\right)$ such that

$$
\lambda(g, g)=\frac{1}{-\epsilon \Delta_{K}(t)}=\frac{-\epsilon}{\Delta_{K}(t)} \in \mathbb{Q}(t) / \mathbb{Z}\left[t^{ \pm 1}\right] .
$$

\subsection{The unknotting number one obstruction by Lickorish}

The following theorem was proved by Lickorish [42] (see also [10, Proposition 2.1]).

Theorem 4.5 Let $K$ be a knot and let $\epsilon \in\{-1,1\}$. If $K$ can be unknotted using one $\epsilon$-crossing change, then there exists a generator $h$ of $H_{1}(\Sigma(K) ; \mathbb{Z})$ such that

$$
l(h, h)=\frac{-2 \epsilon}{\operatorname{det}(K)} \in \mathbb{Q} / \mathbb{Z} .
$$

We will now show that if a knot satisfies the conclusion of Theorem 4.4, then the Lickorish obstruction vanishes. This shows in particular that the Lickorish obstruction gives in fact an obstruction to the algebraic unknotting number being equal to one.

Theorem 4.6 Let $K$ be a knot and let $\eta \in\{-1,1\}$. Suppose there exists a generator $k$ of $H_{1}\left(X(K) ; \mathbb{Z}\left[t^{ \pm 1}\right]\right)$ such that

$$
\lambda(k, k)=\frac{\eta}{\Delta_{K}(t)} \in \mathbb{Q}(t) / \mathbb{Z}\left[t^{ \pm 1}\right] .
$$

Then there exists a generator $h$ of $H_{1}(\Sigma(K) ; \mathbb{Z})$ such that $l(h, h)=\frac{2 \eta}{\operatorname{det}(K)} \in \mathbb{Q} / \mathbb{Z}$.

Proof Suppose there exists a generator $k$ of $H_{1}\left(X(K) ; \mathbb{Z}\left[t^{ \pm 1}\right]\right)$ such that

$$
\lambda(k, k)=\frac{\eta}{\Delta_{K}(t)}=\frac{1}{\eta \Delta_{K}(t)} \in \mathbb{Q}(t) / \mathbb{Z}\left[t^{ \pm 1}\right]
$$

for some $\eta \in\{-1,1\}$. This is equivalent to saying that $\lambda$ is isometric to $\lambda\left(\eta \Delta_{K}(t)\right)$. It follows from Lemma 3.3 that

$$
2 l(K) \cong l(\eta \Delta(-1))=l(\eta \operatorname{det}(K)) \in \mathbb{Q} / \mathbb{Z} .
$$

This means that there exists a generator $g$ for $H_{1}(\Sigma(K) ; \mathbb{Z})$ such that

$$
2 l(g, g)=\frac{\eta}{\operatorname{det}(K)}=\frac{1}{\eta \operatorname{det}(K)} \in \mathbb{Q} / \mathbb{Z} .
$$

Since $\operatorname{det}(K)$ is an odd number, $k=2 g$ is also a generator for $H_{1}(\Sigma(K) ; \mathbb{Z})$, and it is easy to see that it has the required properties. 
Remark (1) We could also easily have deduced Theorem 4.6 from Theorem 3.4. In other words, the Lickorish obstruction is precisely the obstruction of Theorem 3.4 to $n(K)=1$.

(2) Stoimenow [64, page 763 and Conjecture 7.4] conjectures that the Lickorish obstruction contains the obstructions to $u(K)=1$ obtained from the Jones polynomial which was found by Miyazawa [46], Traczyk [67] and Stoimenow [64].

\subsection{The unknotting number one obstructions by Jabuka}

In the following we denote by $W(\mathbb{Q})$ the Witt group of nonsingular bilinear symmetric pairings over $\mathbb{Q}$. Note that we can think of $W(\mathbb{Q})$ also as the Witt group of symmetric matrices over $\mathbb{Q}$ with nonzero determinant. We refer to [23, Section $2 ; 22 ; 56]$ for details. Given a knot $K$ Jabuka [23] denotes by $\varphi(K)$ the element in the Witt group $W(\mathbb{Q})$ defined by $V_{K}+V_{K}^{t}$. The following is now Jabuka's obstruction to $u(K)=1$ :

Theorem 4.7 Let $K$ be a knot and let $\epsilon \in\{-1,1\}$. If $K$ can be unknotted using one $\epsilon$-crossing change, then $\varphi(K)$ is represented by the diagonal matrix with entries $2 \epsilon$ and $-2 \epsilon \operatorname{det}(K)$.

Remark The statement of Theorem 4.7 is precisely the statement of [23, Corollary 1.2]; the only difference is that we view the determinant of a knot as a signed invariant, ie we write $\operatorname{det}(K)=\operatorname{det}\left(V+V^{t}\right)$, whereas Jabuka uses $\left|\operatorname{det}\left(V+V^{t}\right)\right|$ as the definition of the determinant of a knot.

Note that if a knot $K$ can be turned into an Alexander polynomial one knot using one $\epsilon-$ crossing change, then it follows from Theorem 4.1 that $\sigma(K) \in\{0,2 \epsilon\}$. The following result thus shows that the Lickorish obstruction together with the signature obstruction subsumes the Jabuka obstruction.

Theorem 4.8 Let $K$ be a knot and let $\epsilon \in\{-1,1\}$. If $\sigma(K) \in\{0,2 \epsilon\}$ and if there exists a generator $h$ of $H_{1}(\Sigma(K) ; \mathbb{Z})$ such that $l(h, h)=(-2 \epsilon) / \operatorname{det}(K) \in \mathbb{Q} / \mathbb{Z}$, then the conclusion of Theorem 4.7 also holds.

Remark Jabuka [23] showed that in general the Lickorish obstruction is stronger than the obstruction provided by Theorem 4.7, eg the Jabuka obstruction vanishes for $K=8_{8}$, but the Lickorish obstruction detects that $u\left(8_{8}\right) \geq 2$.

In our proof of Theorem 4.8 we will need the following well-known elementary lemma: 
Lemma 4.9 Let $K$ be a knot, then

$$
\operatorname{sign}\left(\Delta_{K}(-1)\right)=(-1)^{\sigma(K) / 2} .
$$

We provide a proof for the reader's convenience.

Proof Let $V$ be a Seifert matrix for $K$. Without loss of generality we can assume $V$ is a $4 k \times 4 k$-matrix. We denote by $p$ the number of positive eigenvalues of $V+V^{t}$ and we denote by $n$ the number of negative eigenvalues of $V+V^{t}$. It follows that

$$
\begin{aligned}
\operatorname{sign}\left(\Delta_{K}(-1)\right) & =\operatorname{sign}\left((-1)^{-2 k} \operatorname{det}\left(-V-V^{t}\right)\right) \\
& =\operatorname{sign}\left(\operatorname{det}\left(V+V^{t}\right)\right)=(-1)^{n}=(-1)^{(n-p) / 2+(n+p) / 2} \\
& =(-1)^{-\sigma(K) / 2} \cdot(-1)^{2 k}=(-1)^{\sigma(K) / 2},
\end{aligned}
$$

completing the proof.

Proof of Theorem 4.8 We will use the notation of the proof of Theorem 4.6. We denote by $W(\mathbb{Z})$ the Witt group of nonsingular pairings over $\mathbb{Z}$. Note that the signature defines an isomorphism

$$
\text { sign: } W(\mathbb{Z}) \rightarrow \mathbb{Z}
$$

We refer to [22] for details. We say that a linking pairing $H \times H \rightarrow \mathbb{Q} / \mathbb{Z}$ is metabolic if there exists a subspace $P \subset H$ with $P=P^{\perp}$. We denote by $W(\mathbb{Z}, \mathbb{Q})$ the Witt group of linking pairings modulo metabolic pairings.

By definition any pairing in $W(\mathbb{Q})$ can be represented by a rational matrix. After multiplying by a sufficiently large square we can also represent a given pairing by an integral symmetric $n \times n$-matrix $A$. To such a matrix we then associate the linking pairing $l(A)$. Note that $l(-A)=-l(A)$ in the group $W(\mathbb{Z}, \mathbb{Q})$. Also note that the above assignment descends to a well-defined map $W(\mathbb{Q}) \rightarrow W(\mathbb{Z}, \mathbb{Q})$ and it is well known that the sequence

$$
0 \rightarrow W(\mathbb{Z}) \rightarrow W(\mathbb{Q}) \rightarrow W(\mathbb{Z}, \mathbb{Q}) \rightarrow 0
$$

is exact. We refer to $[22$, page $90 ; 56]$ for details.

Now let $K$ be a knot and $V$ a Seifert matrix for $K$. Recall (see eg [17]) that the linking pairing $l=l(K)$ is isometric to $l\left(V+V^{t}\right)$. Now suppose we have $\epsilon \in\{-1,1\}$ such that the following hold:

(1) We have $\sigma(K) \in\{0,2 \epsilon\}$.

(2) There exists a generator $h$ of $H_{1}(\Sigma(K)$; $\mathbb{Z})$ such that $l(h, h)=(-2 \epsilon) / d \in \mathbb{Q} / \mathbb{Z}$, where we write $d=\operatorname{det}(K)$. 
We can now prove the following claim.

Claim The element in $W(\mathbb{Q})$ represented by $\left(V+V^{t}\right) \oplus(2 \epsilon / d) \oplus(-2 \epsilon)$ gets sent to the trivial element in $W(\mathbb{Z}, \mathbb{Q})$.

In the following we identify the linking pairing $l(K)$ with the pairing on $\mathbb{Z} / d$ given by $l(a, b)=(-2 \epsilon a b) / d \in \mathbb{Q} / \mathbb{Z}$. Now recall that the image of $\left(V+V^{t}\right) \oplus(2 \epsilon / d) \oplus(-2 \epsilon)$ is represented by the pairing $l(K) \oplus l(2 \epsilon d) \oplus l(-2 \epsilon)$. We consider the map

$$
\begin{aligned}
\mathbb{Z} / 2 \oplus \mathbb{Z} / d & \rightarrow \mathbb{Z} / 2 d, \\
(x, y) & \mapsto x d+2 y .
\end{aligned}
$$

It is straightforward to verify that this map induces an isometry

$$
l(-2 \epsilon) \oplus l(K) \rightarrow l(-2 \epsilon d) .
$$

Put differently, the pairing $l(K) \oplus l(2 \epsilon / d) \oplus l(-2 \epsilon)$ represents the trivial element in $W(\mathbb{Z}, \mathbb{Q})$. This concludes the proof of the claim.

It follows from Lemma 4.9 and from $\sigma(K) \in\{0,2 \epsilon\}$ that the signature of the matrix $\left(V+V^{t}\right) \oplus(2 \epsilon / d) \oplus(-2 \epsilon)$ is zero. It now follows from the claim, the short exact sequence (4-2) and (4-1) that $\left(V+V^{t}\right) \oplus(2 \epsilon / d) \oplus(-2 \epsilon)$ represents the zero element in $W(\mathbb{Q})$. The fact that $(2 \epsilon / d)=(2 \epsilon \cdot d) \in W(\mathbb{Q})$ now completes the proof of the theorem.

\subsection{The unknotting number two obstruction by Stoimenow}

Stoimenow proved the following theorem.

Theorem 4.10 (Stoimenow [64, Theorem 5.2]) Let $K$ be a knot with $|\sigma(K)|=4$ such that $\operatorname{det}(K)$ is a square. If $\operatorname{det}(K)$ has no divisors of the form $4 r+3$, then $u(K)>2$.

Our next theorem shows that the $n(K)$ obstruction contains the Stoimenow obstruction. This shows in particular that the Stoimenow obstruction is an obstruction to the algebraic unknotting number being equal to two. The latter result can also be shown by reading carefully the original proof.

Theorem 4.11 Let $K$ be a knot with $|\sigma(K)|=4$ such that $\operatorname{det}(K)$ is a square. If $n(K)=2$, then $\operatorname{det}(K)$ has a divisor of the form $4 k+3$. 
Proof Let $K$ be a knot with $|\sigma(K)|=4$ and such that $\operatorname{det}(K)$ is a square and suppose $n(K)=2$. Without loss of generality we can assume $\sigma(K)=4$. By Theorem 3.4 there exists a positive-definite matrix $A$ with $|\operatorname{det}(A)|=|\operatorname{det}(K)|$ such that

$$
A=\left(\begin{array}{cc}
4 k+3 & 2 m \\
2 m & 4 l+3
\end{array}\right)
$$

for some $k, l, m \in \mathbb{Z}$. Note that $A$ being positive-definite and $\operatorname{det}(K)$ being a square implies that in fact $\operatorname{det}(A)=\operatorname{det}(K)$.

Since $A$ is positive-definite it also follows that $4 k+3>0$ and that

$$
\operatorname{det}(A)=(4 k+3)(4 l+3)-4 m^{2}
$$

is positive. It follows that $k$ and $l$ actually are positive. Now assume that $\operatorname{det}(A)=$ $\operatorname{det}(K)=d^{2}$ is a square. We thus see that $(4 k+3)(4 l+3)-4 m^{2}=d^{2}$. Since $4 k+3>0$ we can find a prime $p$ of the form $4 r+3$ which divides $4 k+3$. We are done once we show that $p$ also divides $m$.

Suppose that $p$ does not divide $m$. Since $p$ divides $d^{2}+4 m^{2}$ we obtain the equation $d^{2}=-(2 m)^{2} \bmod p$, but since $2 m$ is nonzero, and hence invertible modulo $p$ we obtain that -1 is a square modulo $p$. But it is well known that -1 is not a square modulo a prime of the form $4 r+3$.

\section{New obstructions from the Blanchfield pairing}

\subsection{Obstructions from the Blanchfield pairing to $n(K)=1$}

We have already seen that the Nakanishi index, the signature and the Lickorish criterion give lower bounds on $n(K)$. The Lickorish obstruction can be summarized as replacing an infinite problem (can the Blanchfield pairing be represented by a $1 \times 1-$ matrix over $\mathbb{Z}\left[t^{ \pm 1}\right]$ ) by a finite problem (can the linking pairing be represented by a $1 \times 1$-matrix over $\mathbb{Z}$ ). This principle can easily be generalized.

To formulate the generalizations we need two "reductions" of the Blanchfield pairing. First, let $p$ be a prime. We denote by $Q_{p}$ the quotient field of $\mathbb{F}_{p}\left[t^{ \pm 1}\right]$. Then we can imitate the definition of the Blanchfield pairing over $\mathbb{Z}\left[t^{ \pm 1}\right]$ to define a pairing

$$
H_{1}\left(X(K) ; \mathbb{F}_{p}\left[t^{ \pm 1}\right]\right) \times H_{1}\left(X(K) ; \mathbb{F}_{p}\left[t^{ \pm 1}\right]\right) \rightarrow Q_{p} / \mathbb{F}_{p}\left[t^{ \pm 1}\right] .
$$

Second, let $k$ be an integer such that $H_{1}\left(\Sigma_{k}(K)\right)$ is finite, where $\Sigma_{k}(K)$ denotes the $k$-fold branched cover of $K$. Note that $H_{1}\left(\Sigma_{k}(K)\right)$ is a module over $\mathbb{Z}[\mathbb{Z} / k]$, the group ring of $\mathbb{Z} / k$. We can then define a pairing

$$
H_{1}\left(\Sigma_{k}(K)\right) \times H_{1}\left(\Sigma_{k}(K)\right) \rightarrow S^{-1} \mathbb{Z}[\mathbb{Z} / k] / \mathbb{Z}[\mathbb{Z} / k],
$$


where

$$
S:=\left\{f \in \mathbb{Z}[\mathbb{Z} / k]=\mathbb{Z}\left[t^{ \pm 1}\right] /\left(t^{k}-1\right) \mid f(1)=1\right\} .
$$

The proof that the matrix $A_{K}(t)$ over $\mathbb{Z}\left[t^{ \pm 1}\right]$ (see (2-3)) is a presentation matrix of the Blanchfield pairing over $\mathbb{Z}\left[t^{ \pm 1}\right]$ can also be modified easily to show that $A_{K}(t)$ viewed as a matrix over $\mathbb{F}_{p}\left[t^{ \pm 1}\right]$ respectively over $\mathbb{Z}[\mathbb{Z} / k]=\mathbb{Z}\left[t^{ \pm 1}\right] /\left(t^{k}-1\right)$ is a presentation matrix for the two above pairings. (In particular this shows that both pairings are classical invariants; see also [17] for more information.) We thus obtain the following lemma:

Lemma 5.1 Let $K$ be a knot with $n(K)=n$.

(1) Let $p$ be a prime. Then the $\mathbb{F}_{p}$-Blanchfield pairing

$$
\left.H_{1}\left(X(K) ; \mathbb{F}_{p}\left[t^{ \pm 1}\right]\right) \times H_{1}\left(X(K) ; \mathbb{F}_{p}\left[t^{ \pm 1}\right]\right) \rightarrow Q_{p} / \mathbb{F}_{p}\left[t^{ \pm 1}\right]\right)
$$

can be represented by an $n \times n-$ matrix over $\mathbb{F}_{p}\left[t^{ \pm 1}\right]$.

(2) Let $k$ be an integer; then the $\mathbb{Z}[\mathbb{Z} / k]-$ Blanchfield pairing

$$
H_{1}\left(\Sigma_{k}(K)\right) \times H_{1}\left(\Sigma_{k}(K)\right) \rightarrow S^{-1} \mathbb{Z}[\mathbb{Z} / k] / \mathbb{Z}[\mathbb{Z} / k]
$$

can be represented by an $n \times n-$ matrix over $\mathbb{Z}[\mathbb{Z} / k]$.

If $p$ is any prime, or if $k$ is any integer such that $H_{1}\left(\Sigma_{k}(K) ; \mathbb{Z}\right)$ is finite, then we are dealing with finite objects. In particular in these cases the obstructions provided by the lemma are computable. We implemented both obstructions in the case $n(K)=1$. We applied it to all knots with up to 14 crossings with primes usually up to 11 and $k$ usually up to 6 . (The size of $H_{1}\left(\Sigma_{k}(K)\right)$ typically grows very fast with $k$, putting limitations on the range of $k$.) To our great surprise (and disappointment), among all knots with up to 14 crossings we could not find a single example where these obstructions to $n(K)=1$ could see beyond the Nakanishi index, the Levine-Tristram signatures and the Lickorish obstruction.

In Sections 5.2 and 5.3 we will on the other hand see that using the linking pairing on $H_{1}(\Sigma(K))$ we can give new obstructions to $n(K)=2$ and $n(K)=3$, which actually do work in practice.

\subsection{Obstructions from the linking pairing to $n(K)=2$}

If $K$ is a knot with $n(K)=2$, then it follows from Theorem 3.4 that the linking pairing $l(K)$ can be represented by a certain symmetric $2 \times 2-$ matrix. The full classification of all symmetric $2 \times 2$ matrices up to a congruence was already known to Gauß, below 
we state a slightly weaker result, referring to Conway and Sloane [11, Section 15.3] for an excellent exposition.

Lemma 5.2 Let $A$ be a symmetric integral $2 \times 2-$ matrix with $d:=\operatorname{det}(A) \neq 0$. Then, either $A$ is congruent to a matrix of the form

$$
\left(\begin{array}{ll}
a & c \\
c & b
\end{array}\right)
$$

such that

(1) $0<|a| \leq|b| \leq|d|$,

(2) $c \in\{0, \ldots,\lfloor|a| / 2\rfloor\}$,

or $A$ is congruent to a matrix of the form

$$
\left(\begin{array}{ll}
a & c \\
c & 0
\end{array}\right)
$$

with $c^{2}=d, c \geq 0$ and $a \in\{-c, \ldots, c\}$.

For the reader's convenience we give a short proof of the lemma.

Proof First assume that $A$ is congruent to a matrix such that one of the diagonal entries is zero. It is straightforward to see that in that case $A$ is congruent to a matrix of the latter type.

Now suppose that $A$ is not congruent to matrix such that one of the diagonal entries is zero. Among all matrices congruent to $A$ we then pick a matrix such that the absolute value of the $(1,1)$ entry (ie the top left one) is minimal. We write this matrix as

$$
B:=\left(\begin{array}{ll}
a & c \\
c & b
\end{array}\right) .
$$

After adding a suitable multiple of the first row to the second row and the same multiple of the first column to the second column we can assume that $|c| \leq|a| / 2$. If $c<0$, then we multiply the first row and the first column by minus one, to arrange that $c \geq 0$. By the minimality of $a$, even after these operations, we still have that $|a| \leq|b|$.

Finally note that

$$
\begin{aligned}
|d|=\left|a b-c^{2}\right| & \geq|a||b|-\left(\left\lfloor\frac{|a|}{2}\right\rfloor\right)^{2} \\
& \geq|a||b|-(|a|-1)^{2} \\
& =|b|+(|a|-1)|b|-(|a|-1)^{2}=|b|+(|a|-1)(|b|-|a|+1) \geq|b| .
\end{aligned}
$$

We thus see that $|d| \geq|b|$. 
We can now describe our obstruction to a knot having $n(K)=2$. Let $K$ be a knot with determinant $d=\operatorname{det}(K)$. We denote by $C_{1}, \ldots, C_{l}$ the matrices which satisfy conditions (1) and (2) from the previous corollary applied to $\pm d$, and which are congruent to the identity modulo two. If $\sigma(K)=4 \cdot \epsilon$ for some $\epsilon \in\{-1,1\}$, then we furthermore demand that each $C_{i}$ is $\epsilon$-definite and that each $C_{i}$ is congruent modulo four to a matrix of the form

$$
\left(\begin{array}{cc}
-\epsilon & 2 m \\
2 m & -\epsilon
\end{array}\right)
$$

for some $m \in \mathbb{Z}$. It is clear that this list of matrices $C_{1}, \ldots, C_{l}$ can be explicitly determined.

We can now formulate the following obstruction:

Proposition 5.3 If $n(K)=2$, then there exists an integer $k \in\{1, \ldots, l\}$ and an isometry $l\left(C_{k}\right) \cong 2 l(K)$.

Note that it follows easily from the proof of Theorem 4.11 that this $n(K)=2$ obstruction contains the Stoimenow obstruction.

Proof It is obvious that congruent matrices define isometric linking pairings. The proposition now follows from Theorem 3.4 and Lemma 5.2.

The following lemma gives an elementary way to check whether $2 l(K)$ is isometric to $l(C)$ for a given $2 \times 2-$ matrix $C$ :

Lemma 5.4 Let $C$ be a symmetric integral $2 \times 2-$ matrix with $\operatorname{det}(C)= \pm \operatorname{det}(K)$. Then there exists an isometry $l(C) \cong 2 l(K)$ if and only if there exist $v_{1}, v_{2} \in H_{1}(\Sigma(K))$ which generate $H_{1}(\Sigma(K))$, such that

$$
2 l(K)\left(v_{i}, v_{j}\right)=(i, j) \text {-entry of } C^{-1} \text { modulo } \mathbb{Z}
$$

for any $i, j \in\{1,2\}$.

Proof First let $\Phi: \mathbb{Z}^{2} / C \mathbb{Z}^{2} \rightarrow H_{1}(\Sigma(K))$ be an isomorphism which induces an isometry $l(C) \cong 2 l(K)$. We denote by $v_{1}, v_{2}$ the images of $e_{1}=(1,0)$ and $e_{2}=(0,1)$. It follows immediately from the definitions that $v_{1}$ and $v_{2}$ have the desired properties.

Conversely, suppose we are given $v_{1}, v_{2} \in H_{1}(\Sigma(K))$ which generate $H_{1}(\Sigma(K))$, such that

$$
2 l(K)\left(v_{i}, v_{j}\right)=(i, j) \text {-entry of } C^{-1} \text { modulo } \mathbb{Z}
$$


for any $i, j$. We denote by $\Phi: \mathbb{Z}^{2} \rightarrow H_{1}(\Sigma(K))$ the map given by $\Phi\left(e_{i}\right)=v_{i}$. This map is evidently surjective.

We claim that this map descends to a map $\mathbb{Z}^{2} / C \mathbb{Z}^{2} \rightarrow H_{1}(\Sigma(K))$. Let $v \in C \mathbb{Z}^{2}$. Note that $v^{t} C^{-1} w \in \mathbb{Z}$ for all $w \in \mathbb{Z}^{2}$. It now follows that $l(K)(\Phi(v), \Phi(w))=0 \in \mathbb{Q} / \mathbb{Z}$ for all $w \in \mathbb{Z}^{2}$. But since $\Phi$ is surjective and since $l(K)$ is nondegenerate this implies that $\Phi(v)=0 \in H_{1}(\Sigma(K))$. This shows that $\Phi$ descends to a map $\mathbb{Z}^{2} / C \mathbb{Z}^{2} \rightarrow H_{1}(\Sigma(K))$.

Since the map $\Phi$ is an epimorphism between finite groups of the same size it follows that this map is an isomorphism, and condition (5-1) implies that $\Phi$ is in fact an isometry.

In Section 6.2 we will give several examples of knots where this obstruction applies.

\subsection{Obstructions from the linking pairing to $n(K)=m$ for $m \geq 3$}

The approach in the previous section can also be extended to give an obstruction to $n(K)=m$ for arbitrary $m$. We focus on the definite case (ie when the absolute value of the signature is equal to $2 \mathrm{~m}$ ), and we plan to deal with the general case in a future paper. The key ingredient to getting obstructions is Lemma 5.5 below.

Lemma 5.5 Let $A$ be a positive-definite symmetric $m \times m$-matrix. Then $A$ is congruent to a matrix of the form

$$
\left(\begin{array}{cccc}
f_{11} & f_{12} & \cdots & f_{1 m} \\
f_{21} & f_{22} & \cdots & f_{2 m} \\
\vdots & \vdots & \ddots & \vdots \\
f_{m 1} & f_{m 2} & \cdots & f_{m m}
\end{array}\right)
$$

such that:

(1) $0<f_{11} \leq f_{22} \leq \cdots \leq f_{m m}$.

(2) For any $1 \leq i<j \leq m$ we have $\left|2 f_{i j}\right| \leq f_{i i}$.

(3) $f_{m m} \leq B_{m} \operatorname{det} A$ for a constant $B_{m}$ depending only on $m$.

(4) Furthermore we can take $B_{1}=B_{2}=B_{3}=B_{4}=1$.

Proof By Cassels [8, Theorem 12.1.1] the matrix $A$ can be put into a so-called reduced form (in the sense of Minkowski). Then [8, Lemma 12.1.1] shows parts (1) and (2) of Lemma 5.5. 
To show (3), observe that for a matrix in a reduced form we have

$$
f_{11} \cdot f_{22} \cdots f_{m m} \leq \mu_{m} \operatorname{det} A,
$$

where $\mu_{2}=\frac{4}{3}, \mu_{3}=2, \mu_{4}=4$ and for $m>4$,

$$
\mu_{m}=\left(\frac{2}{\pi}\right)^{m}\left\{\Gamma\left(2+\frac{n}{2}\right)\right\}^{2}\left(\frac{5}{4}\right)^{(n-3)(n-4) / 2},
$$

where $\Gamma$ is the Euler gamma function (see an excellent survey by van der Waerden [70] for proofs and details.) As $f_{i i} \geq 1$, we immediately obtain that $f_{m m} \leq B_{m} \operatorname{det} A$ for $B_{m}=\mu_{m}$.

To show that for $m=2,3,4$ we have $f_{m m} \leq \operatorname{det} A$ we again use (5-2). Let us begin with the case $m=2$. If $f_{11} \geq 2$, by (5-2) we get $f_{22} \leq \frac{2}{3} \operatorname{det} A$. So assume that $f_{11}=1$. Then $f_{12}=f_{21}=0$ because the matrix is in the reduced form (see point (2) in the statement of the lemma). But then $\operatorname{det} A=f_{22}$.

For $m=3$, if $f_{11}>1$ then $f_{11} f_{22} \geq 4$, so $f_{33} \leq \frac{1}{2} \operatorname{det} A$. If $f_{11}=1$, we have $f_{12}=f_{13}=f_{21}=f_{31}=0$, so the form is a block sum of (1) and a two-dimensional form and we use the case $m=2$. The argument with $m=4$ is identical.

If we assume that $f_{i i} \cong 3 \bmod 4$ (cf Theorem 3.4), we can show that $B_{m}$ can be chosen to be 1 for some higher $m$ as well.

Lemma 5.6 If $f_{11}, \ldots, f_{m m}$ are congruent to $-1 \operatorname{modulo} 4$, then $f_{m m} \leq \operatorname{det} A$ for $m \leq 7$.

Proof From the assumptions $f_{i i} \geq 3$, hence by (5-2) we have $f_{m m} \leq 3^{1-m} \mu_{m} \operatorname{det} A$. Now an explicit computation shows that $3^{1-m} \mu_{m} \leq 1$ for $m=5,6,7$.

We can now describe our obstruction to a knot with $|\sigma(K)|=2 m$ having $n(K)=m$. Let $K$ be a knot. We write $d=|\operatorname{det}(K)|$ and $|\sigma(K)|=2 \mathrm{~m}$. Without loss of generality we can assume that $\sigma(K)=2 \mathrm{~m}$. We denote by $C_{1}, \ldots, C_{r}$ the positive-definite $m \times m$-matrices with determinant $d$ which satisfy conditions (1), (2), (3) and (4) from Lemma 5.5, and which are congruent to the identity modulo two. We furthermore restrict ourselves to matrices which are congruent to -1 modulo 4 . It is clear that this list of matrices $C_{1}, \ldots, C_{r}$ can be explicitly determined, even though for large $m$ or $\operatorname{det} A$ this list may be very long.

We can now formulate the following obstruction, which is an immediate consequence of Theorem 3.4 and Lemma 5.5.

Proposition 5.7 If $n(K)=m$, then there exists an integer $s \in\{1, \ldots, r\}$ and an isometry $l\left(C_{S}\right) \rightarrow 2 l(K)$. 
The following lemma gives an elementary way to check whether $2 l(K)$ is isometric to $l(C)$ for a given $m \times m$-matrix $C$ :

Lemma 5.8 Let $C$ be a symmetric $m \times m$-matrix with $\operatorname{det}(C)= \pm \operatorname{det}(K)$. Then there exists an isometry $l(C) \cong 2 l(K)$ if and only if there exist $v_{1}, v_{2}, \ldots, v_{m} \in$ $H_{1}(\Sigma(K))$ which generate $H_{1}(\Sigma(K))$, such that

$$
2 l(K)\left(v_{i}, v_{j}\right)=(i, j) \text {-entry of } C^{-1} \text { modulo } \mathbb{Z}
$$

for any $i, j \in\{1, \ldots, m\}$.

The proof is of course almost identical to the proof of Lemma 5.4. Examples of application of this criterion are given in Section 6.3.

\subsection{Comparison with Owens' obstruction}

We now give a comparison of our obstruction with the Owens obstruction [51, Theorems 1 and 5]. We summarize the key facts:

(1) Owens shows that if a knot satisfies $u(K)=m$ and $|\sigma(K)|=2 m$, then the Heegaard-Floer correction terms (see Ozsváth and Szabó [53]) of the 2-fold branched cover of $K$ satisfy

(a) a certain inequality,

(b) a certain equality modulo 2 .

(2) By work of Ozsváth and Szabó [53, Theorem 1.2] and Taylor [66] (see also [52]) a knot which satisfies the "mod 2 equality" of Owens also satisfies the conclusion of Theorem 3.4.

In practice the fact that one needs to be able to calculate the Heegaard-Floer correction terms of the 2-fold branched cover means that the Owens obstruction can be calculated in a straightforward way for alternating knots, but it is rather difficult to calculate for most other knots. (Note though that calculations can be made if the 2-fold branched cover is a Seifert fibered space; this is the case for Montesinos knots and torus knots.) We conclude with the discussion of some examples:

(1) Owens [51] shows that the $u(K)=2$ obstruction applies to the alternating knots $9_{10}, 9_{13}, 9_{38}, 10_{53}, 10_{101}, 10_{120}$ which have signature equal to four. On the other hand the algebraic unknotting number equals two (using the computer program knotorious we can find explicit algebraic unknotting operations in the sense of [48]). 
(2) Owens furthermore uses Heegaard-Floer homology and a result of Traczyk to show that the unknotting number of the alternating knot $9_{35}$ equals three, even though the signature equals two. Again, the algebraic unknotting number equals two.

(3) Using the obstruction of Section 5.2 we can show that the algebraic unknotting number of the nonalternating knot $11 n_{148}$ equals three, even though the signature equals two. Since the knot is nonalternating and since the signature does not equal four it seems difficult to use the Owens approach to show that the unknotting number equals three.

(4) Owens [51, Corollary 6] also used the $u(K)=3$ obstruction to show that the unknotting number of the two-bridge knot $K=S(51,35)$ equals four. This knot passes our $u_{a}(K)=3$ obstruction, and in fact we can show that the algebraic unknotting number of $K$ equals three.

\section{Examples}

\subsection{Knotorious}

In the following we write

$$
\mathcal{Z}:=\left\{z \in S^{1} \mid z^{1296}=1\right\}
$$

We have written a computer program called knotorious (which is available from the authors' webpages [4]) which given a Seifert matrix calculates the following invariants:

(1) The signature.

(2) The Levine-Tristram signatures $\sigma_{z}(K)$ with $z \in \mathcal{Z}$.

(3) The lower bounds on the Nakanishi index coming from $H_{1}(\Sigma(K))$ and the Alexander module over the finite fields $\mathbb{F}_{2}, \mathbb{F}_{3}, \mathbb{F}_{5}$ and $\mathbb{F}_{7}$.

(4) The Lickorish obstruction.

(5) The Stoimenow obstruction.

(6) The $u(K)=2$ obstruction coming from the linking pairing on $H_{1}(\Sigma(K)$ ) (see Section 5.2).

(7) The $u(K)=3$ obstruction coming from the linking pairing on $H_{1}(\Sigma(K)$ ) (see Section 5.3). 
The program also attempts to compute, in a nonrigorous way, the invariant $\mu(K)$. Furthermore, the program also finds upper bounds on the algebraic unknotting number by finding explicit algebraic unknotting moves (we refer to [48; 60, Section 2] for details on algebraic unknotting moves). We calculated the above invariants for all knots with up to 12 crossings, the details can be found on the authors' webpages [4]. All the examples in the subsequent sections are based on the calculations with knotorious.

\subsection{Examples for the new $n(K)=2$ obstruction coming from $l(K)$}

Let $K$ be a knot. In Section 5.2 we showed that the linking from $l(K)$ on the homology of the 2 -fold branched cover of $K$ gives an obstruction to $u(K) \leq 2$. We applied this obstruction to all knots with up to 12 crossings. We found that the following knots with $|\sigma(K)|=4$ (in fact with $\mu(K)=4$ ) and $m(K) \leq 2$ have $n(K)>2$ :

$\begin{array}{rllllll}9_{49} & 11 a_{123} & 11 n_{133} & 12 a_{311} & 12 a_{386} & 12 a_{433} & 12 a_{561} \\ 12 a_{563} & 12 a_{569} & 12 a_{664} & 12 a_{683} & 12 a_{725} & 12 a_{780} & 12 a_{907} \\ & 12 n_{276} & 12 n_{494} & 12 n_{496} & 12 n_{626} & 12 n_{654} & \end{array}$

Furthermore the following knots have $|\sigma(K)| \leq 2$ (in fact $\mu(K) \leq 2$ ) and $m(K) \leq 2$ but $n(K)>2$ :

$$
10_{103} \quad 11 n_{148} \quad 12 a_{327} \quad 12 a_{921} \quad 12 a_{1194} \quad 12 n_{147}
$$

We now discuss to what degree previous invariants detect the unknotting numbers of the above examples:

(1) The Stoimenow obstruction applies to $9_{49}, 11 n_{133}, 12 a_{664}$ and $12 n_{276}$, but does not apply to any of the other knots. To the best of our knowledge none of the other previous classical invariants detect that $n(K)>1$.

(2) Stoimenow [64] also used the Brandt-Lickorish-Millett-Ho polynomial (see Ho [21] and Brandt, Lickorish and Millett [7]) to give an obstruction to $u(K)=2$. Stoimenow shows that this criterion implies that $u\left(10_{103}\right)>2$. We did not check this criterion for the above 12 crossing knots.

(3) Note that most of the above knots (including $10_{103}$ but not $9_{49}$ ) are alternating, in that case the Rasmussen $s$-invariant and the Ozsváth-Szabó $\tau$-invariant agree (up to a scaling factor) with the signature, in particular they do not determine the unknotting number of the above 12 alternating knots.

(4) The $s$-invariant has been computed for all knots with up to 12 crossings (see [9]), it detects the unknotting number for only one of the above nonalternating knots, namely $12_{n 276}$. 
(5) The $\tau$-invariant has been calculated for all knots with up to 11 crossings by Baldwin and Gillam (see [1]), it does not detect the unknotting number for any of the above nonalternating knots with up to 11 crossings.

(6) Arguably $11 n_{148}$ is the most interesting example. Many invariants for nonalternating knots are very difficult to calculate (eg the Heegaard-Floer correction terms of the 2 -fold branched cover, as in $[51 ; 55])$. The aforementioned calculations show that the $\tau$-invariant and the $s$-invariant do not detect the unknotting number of $11 n_{148}$. Furthermore, several obstructions to $u(K)=2($ eg $[51 ; 64])$ can be applied only if $|\sigma(K)|=4$, whereas we have $\sigma\left(11 n_{148}\right)=2$.

The webpage knotinfo [9], maintained by Cha and Livingston, collects the unknotting information on knots up to eleven crossings. According to this information it was not known before these calculations that $u\left(11 a_{123}\right)=3$ and $u\left(11 n_{148}\right)=3$.

\subsection{Examples for the $n(K)=3$ obstruction coming from $l(K)$}

Let $K$ be a knot. In Section 5.3 we showed that the linking from $l(K)$ gives rise to an obstruction to $u(K) \leq 3$. We applied this obstruction to all knots with up to 14 crossings with $|\sigma(K)|=6$ and found that it applies to precisely two knots, namely $14 n_{12777}$ and $14 a_{4637}$.

We expect that given any $m \in \mathbb{N}$ there exist examples of knots such that the obstruction introduced in Section 5.3 detects that $u_{a}(K)=m$, but such that all previous classical lower bounds do not detect that $u_{a}(K)=m$. Finding such examples will obviously require a different method than the brute force search we have done with knotorious.

\subsection{Knots with up to 11 crossings}

Our calculations with knotorious show that the Nakanishi index, the signature, the Lickorish obstruction and the $n(K)=2$ obstruction of Section 5.2 completely determine the algebraic unknotting number for all knots with up to 11 crossings.

The full details are available in [4]. For the reader's convenience we provide in Table 1 the algebraic unknotting number for all knots with up to 10 crossings. The subscripts denote the way of obtaining the result:

- The notation $1_{u}$ means that there exists a single algebraic unknotting move which changes the knot into a knot with trivial Alexander polynomial.

- The notation $2_{L}$ means that we use the Lickorish obstruction. 
- The notation $k_{\sigma}$ for $k=2,3,4$ means that the signature detects the algebraic unknotting number and it is greater than one.

- The notation 2 s stands for the Wendt criterion, in particular the Nakanishi index is equal to two.

- The notation $2 A$ means that the minimal number of generators of the Alexander module over $\mathbb{F}_{2}\left[t^{ \pm}\right]$is two.

- The notation $3{ }_{S}$ means that the Stoimenow obstruction (see Section 4.7) applies.

- The notation $\mathbf{3}_{\boldsymbol{n}}$ denotes our new obstruction as discussed in Section 5.2.

(Note that in some cases two or more obstructions will detect $u_{a}(K)$, but we will only indicate one obstruction.)

The star indicates that the unknotting number is actually known (according to [9]) to be larger than $u_{a}(K)$. We remark that for knots $10_{61}, 10_{76}$ and $10_{100}$, we computed that $u_{a}(K)=2$, but it is not known, whether $u(K)=2$ or 3 . These knots are not marked by a star.

\subsection{Knots with 12 crossings}

Among the 12 crossing knots we found the following examples:

(1) There exists precisely one knot, namely $12 n_{749}$ with $|\sigma(K)| \leq 2$ and such that $\left|\sigma_{z}(K)\right| \geq 4$ for some $z \in \mathcal{Z}$.

(2) There exists precisely one knot, namely $12 a_{896}$ with $\left|\sigma_{z}(K)\right| \leq 2$ for all $z \in \mathcal{Z}$ and such that there exist $z_{1}, z_{2} \in \mathcal{Z}$ with $\left|\sigma_{z_{1}}(K)-\sigma_{z_{2}}(K)\right|=4$.

Our calculations show that the aforementioned seven lower bounds determine the algebraic unknotting number for all knots with up to 12 crossings, except possibly for the following:

$\begin{array}{lllllll}12 a_{0050} & 12 a_{0141} & 12 a_{0364} & 12 a_{0649} & 12 a_{0728} & 12 a_{0791} & 12 a_{0901} \\ 12 a_{1049} & 12 a_{1054} & 12 a_{1064} & 12 a_{1138} & 12 a_{1141} & 12 a_{1234} & 12 a_{1236} \\ & 12 a_{1264} & 12 n_{0200} & 12 n_{0260} & 12 n_{0657} & 12 n_{0864} & \end{array}$

The algebraic unknotting number of all the above knots is either 1 or 2 .

The knots

$$
\begin{array}{lllllll}
12 a_{0050} & 12 a_{0141} & 12 a_{0364} & 12 a_{0649} & 12 a_{0728} & 12 a_{0791} & 12 a_{0901} \\
12 a_{1054} & 12 a_{1064} & 12 a_{1138} & 12 a_{1234} & 12 a_{1236} & 12 n_{0200} & 12 n_{0864}
\end{array}
$$




\begin{tabular}{|c|c|c|c|c|c|c|c|c|c|c|c|c|c|}
\hline not & $n$ & not & $n$ & not & $n$ & not & $n$ & not & $n$ & knot & $n$ & not & $n$ \\
\hline $3_{1}$ & $x_{0}$ & 2 & $\mathrm{I}_{u}$ & & & & $2 \sigma$ & 61 & $2 \sigma$ & $10_{97}$ & $L$ & $0_{133}$ & $1_{u}$ \\
\hline $4_{1}$ & $1_{u}$ & $9_{3}$ & $3_{\sigma}$ & 39 & $1_{u}$ & $0_{26}$ & $1_{u}$ & $0_{62}$ & $2_{\sigma}$ & $10_{98}$ & $2_{w}$ & $10_{134}$ & $3_{\sigma}$ \\
\hline $5_{1}$ & $2 \sigma$ & $9_{4}$ & $2_{\sigma}$ & $9_{40}$ & $2 w$ & $10_{27}$ & $1_{u}$ & $10_{63}$ & $2_{\sigma}$ & $10_{99}$ & $2_{w}$ & $10_{135}$ & $1_{u}^{*}$ \\
\hline $5_{2}$ & $1_{u}$ & $9_{5}$ & $1_{u}^{*}$ & $9_{41}$ & $2{ }_{w}$ & $10_{28}$ & $1_{u}^{*}$ & $10_{64}$ & $1_{u}^{*}$ & $10_{100}$ & $2_{\sigma}$ & $10_{136}$ & $1_{u}$ \\
\hline $6_{1}$ & $1_{u}$ & $9_{6}$ & $3_{\sigma}$ & $9_{42}$ & $1_{u}$ & $10_{29}$ & $2_{L}$ & $10_{65}$ & $2_{L}$ & $10_{101}$ & & & $1_{u}$ \\
\hline $6_{2}$ & $1_{u}$ & $9_{7}$ & $2_{\sigma}$ & & $2_{\sigma}$ & & $1_{u}$ & & & $10_{102}$ & & & $1_{u}^{*}$ \\
\hline $6_{3}$ & $1_{u}$ & $9_{8}$ & $1_{u}^{*}$ & $9_{44}$ & $1_{u}$ & $10_{31}$ & $1_{u}$ & $10_{67}$ & ${ }^{2} L$ & $10_{103}$ & $3_{n}$ & $10_{139}$ & $3_{\sigma}^{*}$ \\
\hline $7_{1}$ & $3_{\sigma}$ & $9_{9}$ & $3_{\sigma}$ & $9_{45}$ & $1_{u}$ & $0_{32}$ & $1_{u}$ & $0_{68}$ & $1_{u}^{*}$ & $10_{104}$ & $1_{u}$ & $10_{140}$ & $2_{A}$ \\
\hline $7_{2}$ & $1_{u}$ & $9_{10}$ & $2_{\sigma}^{*}$ & $9_{46}$ & $2{ }_{w}$ & $10_{33}$ & $1_{u}$ & $10_{69}$ & $2_{L}$ & $10_{105}$ & $2_{L}$ & $10_{141}$ & $1_{u}$ \\
\hline $7_{3}$ & $2_{\sigma}$ & $9_{11}$ & $2_{\sigma}$ & $9_{47}$ & $2{ }_{w}$ & & $1_{u}^{*}$ & & & & $2_{L}$ & & $3_{\sigma}$ \\
\hline 74 & $2_{L}$ & $9_{12}$ & $1_{u}$ & & & & $1_{u}^{*}$ & & & & & & $1_{u}$ \\
\hline 75 & $2_{\sigma}$ & $9_{13}$ & $2_{\sigma}^{*}$ & $9_{49}$ & $3 S$ & $10_{36}$ & $2_{L}$ & $0_{72}$ & $2_{\sigma}$ & $10_{108}$ & $2_{L}$ & $10_{144}$ & $2_{L}$ \\
\hline 76 & $1_{u}$ & $9_{14}$ & $1_{u}$ & $10_{1}$ & $1_{u}$ & $10_{37}$ & $1_{u}^{*}$ & $0_{73}$ & $1_{u}$ & $10_{109}$ & $2_{L}$ & & $1_{u}^{*}$ \\
\hline $7_{7}$ & $1_{u}$ & $9_{15}$ & $2_{L}$ & $10_{2}$ & $3_{\sigma}$ & $10_{38}$ & $1_{u}^{*}$ & $0_{74}$ & $2_{w}$ & $10_{110}$ & & & $1_{u}^{u}$ \\
\hline $8_{1}$ & $1_{u}$ & $9_{16}$ & $3_{\sigma}$ & & $2_{L}$ & & $2_{\sigma}$ & & & & & & $1_{u}$ \\
\hline $8_{2}$ & $2_{\sigma}$ & $9_{17}$ & $2_{L}$ & & $1_{u}^{*}$ & & $2_{L}$ & & $2_{\sigma}$ & & & & $1_{u}^{*}$ \\
\hline $8_{3}$ & $1_{u}^{*}$ & $9_{18}$ & $2_{\sigma}$ & $10_{5}$ & $2_{\sigma}$ & $10_{41}$ & $1_{u}^{*}$ & $0_{77}$ & $1_{u}^{*}$ & $10_{113}$ & $1_{u}$ & & $2_{\sigma}$ \\
\hline 84 & $1_{u}^{*}$ & $9_{19}$ & $1_{u}$ & $10_{6}$ & $2_{\sigma}^{*}$ & $10_{42}$ & $1_{u}$ & $0_{78}$ & $2_{\sigma}$ & $10_{114}$ & $1_{u}$ & $10_{150}$ & $2_{\sigma}$ \\
\hline $8_{5}$ & $2_{\sigma}$ & $9_{20}$ & $2_{\sigma}$ & $10_{7}$ & $1_{u}$ & $10_{43}$ & $1_{u}^{*}$ & $0_{79}$ & $1_{u}^{*}$ & $10_{115}$ & $2_{A}$ & $10_{151}$ & $1_{u}^{*}$ \\
\hline $8_{6}$ & $1_{u}^{*}$ & $9_{21}$ & $1_{u}$ & $10_{8}$ & $2_{\sigma}$ & $10_{44}$ & $1_{u}$ & $10_{80}$ & $3_{\sigma}$ & $10_{116}$ & $2_{L}$ & & $3_{\sigma}^{*}$ \\
\hline $8_{7}$ & $1_{u}$ & $9_{22}$ & $1_{u}$ & $10_{9}$ & $1_{u}$ & $10_{45}$ & $1_{u}^{*}$ & $10_{81}$ & $1_{u}^{*}$ & & $1_{u}^{*}$ & & $1_{u}^{*}$ \\
\hline $8_{8}$ & $2_{L}$ & $9_{23}$ & $2_{\sigma}$ & $10_{10}$ & $1_{u}$ & $10_{46}$ & $3_{\sigma}$ & 82 & $1_{u}$ & & $1_{u}$ & & $2_{\sigma}^{*}$ \\
\hline 89 & $1_{u}$ & $9_{24}$ & $1_{u}$ & $10_{11}$ & $1_{u}^{*}$ & $10_{47}$ & $2_{\sigma}$ & $10_{83}$ & $1_{u}^{*}$ & $10_{119}$ & $1_{u}$ & & $2 w$ \\
\hline $8_{10}$ & $1_{u}^{*}$ & $9_{25}$ & $1_{u}^{*}$ & $10_{12}$ & $1_{u}^{*}$ & $10_{48}$ & $1_{u}^{*}$ & $10_{84}$ & $1_{u}$ & $10_{120}$ & $2_{\sigma}^{*}$ & $10_{156}$ & $1_{u}$ \\
\hline $8_{11}$ & $1_{u}$ & $9_{26}$ & $1_{u}$ & $10_{13}$ & $1_{u}^{*}$ & $10_{49}$ & $3_{\sigma}$ & $10_{85}$ & $2_{\sigma}$ & $10_{121}$ & $2_{L}$ & $10_{157}$ & $2_{w}$ \\
\hline $8_{12}$ & $1_{u}^{*}$ & $9_{27}$ & $1_{u}$ & $10_{14}$ & $2_{\sigma}$ & $10_{50}$ & $2_{\sigma}$ & $10_{86}$ & $2_{L}$ & $10_{122}$ & $2_{L}$ & & $1_{u}^{*}$ \\
\hline $8_{13}$ & $1_{u}$ & $9_{28}$ & $1_{u}$ & & $1_{u}^{*}$ & $10_{51}$ & $1_{u}^{*}$ & & $1_{u}^{*}$ & & $2_{w}$ & & $1_{u}$ \\
\hline $8_{14}$ & $1_{u}$ & $9_{29}$ & $1_{u}^{*}$ & $10_{16}$ & $1_{u}^{*}$ & & $1_{u}^{*}$ & $10_{88}$ & $1_{u}$ & $10_{124}$ & $4_{\sigma}$ & 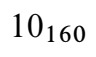 & $2_{\sigma}$ \\
\hline $8_{15}$ & $2_{\sigma}$ & $9_{30}$ & $1_{u}$ & & $1_{u}$ & $10_{53}$ & $2_{\sigma}^{*}$ & $10_{89}$ & $2_{L}$ & $10_{125}$ & $1_{u}^{*}$ & & $2_{\sigma}^{*}$ \\
\hline $8_{16}$ & $2_{L}$ & $9_{31}$ & $2_{L}$ & $10_{18}$ & $1_{u}$ & $10_{54}$ & $1_{u}^{*}$ & $10_{90}$ & $1_{u}^{*}$ & $10_{126}$ & $1_{u}^{*}$ & $10_{162}$ & $1_{u}^{*}$ \\
\hline $8_{17}$ & $1_{u}$ & $9_{32}$ & $1_{u}^{*}$ & $10_{19}$ & $2_{L}$ & $10_{55}$ & $2_{\sigma}$ & $10_{91}$ & $1_{u}$ & $10_{127}$ & $2_{\sigma}$ & & $2_{L}$ \\
\hline $8_{18}$ & $2 w$ & $9_{33}$ & $1_{u}$ & & $2_{L}$ & $10_{56}$ & $2_{\sigma}$ & & $2_{\sigma}$ & & $3_{\sigma}$ & & $1_{u}$ \\
\hline $8_{19}$ & $3_{\sigma}$ & $9_{34}$ & $1_{u}$ & $10_{21}$ & $2_{\sigma}$ & $10_{57}$ & $1_{u}^{*}$ & $10_{93}$ & $1_{u}^{*}$ & $10_{129}$ & $1_{u}$ & $10_{165}$ & $2_{L}$ \\
\hline $8_{20}$ & $1_{u}$ & $9_{35}$ & $2_{w}^{*}$ & & $1_{u}^{*}$ & & $1_{u}^{*}$ & $10_{94}$ & $1_{u}^{*}$ & $10_{130}$ & & & \\
\hline $8_{21}$ & $1_{u}$ & $9_{36}$ & $2_{\sigma}$ & $10_{23}$ & $1_{u}$ & $10_{59}$ & $1_{u}$ & 95 & $1_{u}$ & $10_{131}$ & $1_{u}$ & & \\
\hline $9_{1}$ & $4_{\sigma}$ & $9_{37}$ & $2 w$ & $10_{24}$ & $2_{L}$ & $10_{60}$ & $1_{u}$ & $0_{96}$ & $1_{u}^{*}$ & $10_{132}$ & $1_{u}$ & & \\
\hline
\end{tabular}

Table 1: Algebraic unknotting number for knots with up to 10 crossings; see Section 6.4 for an explanation of symbols. 
all have Nakanishi index 1. In fact, we were able to find an explicit generator of the Alexander module. This allows us to compute the Blanchfield pairing for all those knots. Since $m(K)=1$ it is necessarily of the form

$$
\Lambda / p \Lambda \times \Lambda / p \Lambda \stackrel{(v, w) \mapsto \bar{v} \cdot q \cdot w / p}{\longrightarrow} \Omega / \Lambda,
$$

where $p$ is the Alexander polynomial and $q \in \Lambda$. For example, for $K=12 a_{0050}$,

$$
\begin{aligned}
& p=\Delta_{K}(t)=t^{-4}-8 t^{-3}+20 t^{-2}-30 t^{-1}+33 t-30 t+20 t^{2}-8 t^{3}+t^{4}, \\
& q=-t^{3}+7 t^{2}-13 t+17-13 t^{-1}+7 t^{-2}-t^{-3} ;
\end{aligned}
$$

we refer to [4] for the other knots. Thus $12 a_{0050}$ has algebraic unknotting number 1 if and only if there exists an automorphism of $\Lambda / p$ (as a $\Lambda$-module), which transforms this pairing into $(v, w) \mapsto \pm \bar{v} w / p$. This is equivalent to the existence of an $f \in \Lambda$ such that $q f \bar{f}= \pm 1(\bmod p)$. The problem of finding such $f$ or showing that it does not exist, in general, is very hard.

The knots $12 a_{1054}, 12 a_{1141}, 12 a_{1264}, 12 n_{657}$ and $12 n_{0260}$ have Nakanishi index 1 or 2 . We were able to find a $2 \times 2$ presentation matrix in each case. For example, for $12 a_{1054}$ we have

$$
\left(\begin{array}{cc}
2 t^{3} & -1+4 t-7 t^{2}+4 t^{3}-t^{4} \\
1-5 t+t^{2}-5 t^{3}+t^{4} & 3 t
\end{array}\right) .
$$

However, we could not show that the Alexander module is cyclic. If it is not, then the algebraic unknotting number is 2 .

\section{Open questions}

Apart from Conjecture 1.4, we state a few more questions and problems related to $n(K)$.

Question 1 Given any knot $K$, do we have the following equality:

$$
n(K)=\text { minimal size of a hermitian matrix } A \text { over } \mathbb{Z}\left[t^{ \pm 1}\right] \text { with } \lambda(A) \cong \lambda(K) \text { ? }
$$

Put differently, is the condition in the definition of $n(K)$ that $A(1)$ be diagonal over $\mathbb{Z}$ necessary? Note that an affirmative answer would imply that $n(K) \leq \operatorname{deg} \Delta_{K}(t)$ for any knot $K$. Added in proof: The question was answered in the affirmative in [5].

Question 2 Let $K$ be a knot with $m(K)=1,|\sigma(K)| \leq 2$ satisfying the Lickorish obstruction. Does it follow that the $n(K)=1$ obstructions of Lemma 5.1 are necessarily satisfied? The discussion following Lemma 5.1 is evidence that the answer is yes. 
Question 3 Is $n(K)$ invariant under mutation? It is an open question whether the unknotting number is preserved under mutation (see [33, Problem 1.69(c)]). The $S$-equivalence class of a Seifert matrix (and thus the isometry type of the Blanchfield pairing) is preserved under positive mutation (see Kirk and Livingston [34, Theorem 2.1]). On the other hand the $S$-equivalence class (in fact the isomorphism class of the Alexander module) is in general not preserved under negative mutation (see Kearton [28; 29, Section 3]). We do not know whether $n(K)$ is preserved under mutation. Note though that the Levine-Tristram signatures are preserved under any mutation (see Cooper and Lickorish [12]) and note that homeomorphism type of the 2-fold branched cover is preserved under mutation (see eg [26, Proposition 3.8.2]).

Question 4 In Section 4.3 we introduce a new classical invariant $\rho_{\mathbb{Z}\left[t^{ \pm 1}\right]}(K)$ and we show in Proposition 4.2 that it gives a lower bound on the topological 4-genus. Can this invariant be used to give new computable lower bounds on the topological 4-genus?

Question 5 What are the algebraic unknotting numbers of the remaining 12 crossing knots? (See Section 6.5 for details.) It might be possible to possible to use the methods of Kearton and Wilson [30] to show that the Nakanishi index of the five 12 crossing knots mentioned in Section 6.5 is 2.

\section{References}

[1] J A Baldwin, W D Gillam, Computations of Heegaard-Floer knot homology, J. Knot Theory Ramifications 21 (2012) 1250075, 65 MR2925428

[2] R C Blanchfield, Intersection theory of manifolds with operators with applications to knot theory, Ann. of Math. 65 (1957) 340-356 MR0085512

[3] M Boileau, C Weber, Le problème de J Milnor sur le nombre gordien des nouds algébriques, Enseign. Math. 30 (1984) 173-222 MR767901

[4] M Borodzik, S Friedl, Knotorious world wide web page Available at http:// www.mimuw.edu.pl/ mcboro/knotorious.php

[5] M Borodzik, S Friedl, On the algebraic unknotting number, Trans. Lon. Math. Soc. 1 (2014) 57-84

[6] M Borodzik, S Friedl, The unknotting number and classical invariants, II, Glasg. Math. J. 56 (2014) 657-680 MR3250270

[7] R D Brandt, W B R Lickorish, K C Millett, A polynomial invariant for unoriented knots and links, Invent. Math. 84 (1986) 563-573 MR837528

[8] J W S Cassels, Rational quadratic forms, London Math. Soc. Monographs 13, Academic Press, New York (1978) MR522835 
[9] J C Cha, C Livingston, KnotInfo: Table of knot invariants Available at http:// www. indiana. edu/ knotinfo

[10] T D Cochran, W B R Lickorish, Unknotting information from 4-manifolds, Trans. Amer. Math. Soc. 297 (1986) 125-142 MR849471

[11] J H Conway, N J A Sloane, Sphere packings, lattices and groups, 3rd edition, Grundl. Math. Wissen. 290, Springer, New York (1999) MR1662447

[12] D Cooper, W B R Lickorish, Mutations of links in genus-2 handlebodies, Proc. Amer. Math. Soc. 127 (1999) 309-314 MR1605940

[13] M E Fogel, The algebraic unknotting number, PhD thesis, UC Berkeley (1993) Available at http://search.proquest.com/docview/304077909

[14] M E Fogel, Knots with algebraic unknotting number one, Pacific J. Math. 163 (1994) 277-295 MR1262298

[15] M H Freedman, F Quinn, Topology of 4-manifolds, Princeton Math. Series 39, Princeton Univ. Press (1990) MR1201584

[16] S Friedl, I Hambleton, P Melvin, P Teichner, Nonsmoothable four-manifolds with infinite cyclic fundamental group, Int. Math. Res. Not. 2007 (2007) MR2344268

[17] C M Gordon, Some aspects of classical knot theory, from: "Knot theory", (J-C Hausmann, editor), Lecture Notes in Math. 685, Springer, Berlin (1978) 1-60 MR521730

[18] CM Gordon, J Luecke, Knots with unknotting number 1 and essential Conway spheres, Algebr. Geom. Topol. 6 (2006) 2051-2116 MR2263059

[19] J E Greene, Donaldson's theorem, Heegaard-Floer homology, and knots with unknotting number one, Adv. Math. 255 (2014) 672-705 MR3167496

[20] J Hillman, Algebraic invariants of links, 2nd edition, Series on Knots and Everything 52, World Scientific, River Edge, NJ (2012) MR2931688

[21] C F Ho, A polynomial invariant for knots and links: Preliminary report (1985)

[22] D Husemoller, J Milnor, Symmetric bilinear forms, Springer, New York (1973) MR0506372

[23] S Jabuka, The rational Witt class and the unknotting number of a knot, Preprint (2009)

[24] T Kanenobu, H Murakami, Two-bridge knots with unknotting number one, Proc. Amer. Math. Soc. 98 (1986) 499-502 MR857949

[25] A Kawauchi, Three dualities on the integral homology of infinite cyclic coverings of manifolds, Osaka J. Math. 23 (1986) 633-651 MR866269

[26] A Kawauchi, A survey of knot theory, Birkhäuser, Basel (1996) MR1417494

[27] C Kearton, Blanchfield duality and simple knots, Trans. Amer. Math. Soc. 202 (1975) 141-160 MR0358796

[28] C Kearton, Mutation of knots, Proc. Amer. Math. Soc. 105 (1989) 206-208 MR929430 
[29] C Kearton, S-equivalence of knots, J. Knot Theory Ramifications 13 (2004) 709-717 MR2088740

[30] C Kearton, S M J Wilson, Knot modules and the Nakanishi index, Proc. Amer. Math. Soc. 131 (2003) 655-663 MR1933359

[31] S Kinoshita, On Wendt's theorem of knots, Osaka Math. J. 9 (1957) 61-66 MR0090054

[32] S Kinoshita, On Wendt's theorem of knots, II, Osaka Math. J. 10 (1958) 259-261 MR0124889

[33] R Kirby, Problems in low-dimensional topology, from: "Geometric topology", (W H Kazez, editor), AMS/IP Stud. Adv. Math. 2, Amer. Math. Soc. (1997) 35-473 MR1470751

[34] P Kirk, C Livingston, Concordance and mutation, Geom. Topol. 5 (2001) 831-883 MR1871406

[35] K H Ko, A Seifert-matrix interpretation of Cappell and Shaneson's approach to link cobordisms, Math. Proc. Cambridge Philos. Soc. 106 (1989) 531-545 MR1010376

[36] T Kobayashi, Minimal genus Seifert surfaces for unknotting number 1 knots, Kobe J. Math. 6 (1989) 53-62 MR1023526

[37] P B Kronheimer, T S Mrowka, Gauge theory for embedded surfaces, I, Topology 32 (1993) 773-826 MR1241873

[38] C Leidy, Higher-order linking forms for knots, Comment. Math. Helv. 81 (2006) 755-781 MR2271220

[39] J Levine, Knot cobordism groups in codimension two, Comment. Math. Helv. 44 (1969) 229-244 MR0246314

[40] J Levine, An algebraic classification of some knots of codimension two, Comment. Math. Helv. 45 (1970) 185-198 MR0266226

[41] J Levine, Knot modules, I, Trans. Amer. Math. Soc. 229 (1977) 1-50 MR0461518

[42] W B R Lickorish, The unknotting number of a classical knot, from: "Combinatorial methods in topology and algebraic geometry”, (J R Harper, R Mandelbaum, editors), Contemp. Math. 44, Amer. Math. Soc. (1985) 117-121 MR813107

[43] W B R Lickorish, An introduction to knot theory, Graduate Texts in Math. 175, Springer, New York (1997) MR1472978

[44] C Livingston, Knot 4-genus and the rank of classes in $W(\mathbb{Q}(t))$, Pacific J. Math. 252 (2011) 113-126 MR2862144

[45] J Ma, R Qiu, A lower bound on unknotting number, Chinese Ann. Math. Ser. B 27 (2006) 437-440 MR2250868

[46] Y Miyazawa, The Jones polynomial of an unknotting number one knot, Topol. Appl. 83 (1998) 161-167 MR1606374

[47] J M Montesinos, Seifert manifolds that are ramified two-sheeted cyclic coverings, Bol. Soc. Mat. Mexicana 18 (1973) 1-32 MR0341467 
[48] H Murakami, Algebraic unknotting operation, from: "Proceedings of the Second Soviet-Japan Joint Symposium of Topology”, Questions Answers Gen. Topol. 8 (1990) 283-292 MR1043226

[49] K Murasugi, On a certain numerical invariant of link types, Trans. Amer. Math. Soc. 117 (1965) 387-422 MR0171275

[50] Y Nakanishi, A note on unknotting number, Math. Sem. Notes Kobe Univ. 9 (1981) 99-108 MR634000

[51] B Owens, Unknotting information from Heegaard Floer homology, Adv. Math. 217 (2008) 2353-2376 MR2388097

[52] B Owens, S Strle, Definite manifolds bounded by rational homology three spheres, from: "Geometry and topology of manifolds", (H U Boden, I Hambleton, A J Nicas, B D Park, editors), Fields Inst. Commun. 47, Amer. Math. Soc. (2005) 243-252 MR2189936

[53] P Ozsváth, Z Szabó, Absolutely graded Floer homologies and intersection forms for four-manifolds with boundary, Adv. Math. 173 (2003) 179-261 MR1957829

[54] P Ozsváth, Z Szabó, Knot Floer homology and the four-ball genus, Geom. Topol. 7 (2003) 615-639 MR2026543

[55] P Ozsváth, Z Szabó, Knots with unknotting number one and Heegaard Floer homology, Topology 44 (2005) 705-745 MR2136532

[56] A Ranicki, Exact sequences in the algebraic theory of surgery, Math. Notes 26, Princeton Univ. Press (1981) MR620795

[57] A Ranicki, Blanchfield and Seifert algebra in high-dimensional knot theory, Mosc. Math. J. 3 (2003) 1333-1367 MR2058802

[58] J A Rasmussen, Floer homology and knot complements, PhD thesis, Harvard University (2003) Available at http://search.proquest.com/docview/305332635

[59] J Rasmussen, Khovanov homology and the slice genus, Invent. Math. 182 (2010) 419-447 MR2729272

[60] O Saeki, On algebraic unknotting numbers of knots, Tokyo J. Math. 22 (1999) 425-443 MR1727885

[61] S Sarkar, Grid diagrams and the Ozsváth-Szabó tau-invariant, Math. Res. Lett. 18 (2011) 1239-1257 MR2915478

[62] M G Scharlemann, Unknotting number one knots are prime, Invent. Math. 82 (1985) 37-55 MR808108

[63] M G Scharlemann, A Thompson, Link genus and the Conway moves, Comment. Math. Helv. 64 (1989) 527-535 MR1022995

[64] A Stoimenow, Polynomial values, the linking form and unknotting numbers, Math. Res. Lett. 11 (2004) 755-769 MR2106240 
[65] L R Taylor, On the genera of knots, from: "Topology of low-dimensional manifolds", (R A Fenn, editor), Lecture Notes in Math. 722, Springer, Berlin (1979) 144-154 MR547461

[66] L R Taylor, Relative Rochlin invariants, Topol. Appl. 18 (1984) 259-280 MR769295

[67] P Traczyk, A criterion for signed unknotting number, from: "Low-dimensional topology”, (H Nencka, editor), Contemp. Math. 233, Amer. Math. Soc. (1999) 215-220 MR1701685

[68] A G Tristram, Some cobordism invariants for links, Proc. Cambridge Philos. Soc. 66 (1969) 251-264 MR0248854

[69] H F Trotter, On S-equivalence of Seifert matrices, Invent. Math. 20 (1973) 173-207 MR0645546

[70] B L van der Waerden, Die Reduktionstheorie der positiven quadratischen Formen, Acta Math. 96 (1956) 265-309 MR0082513

[71] H Wendt, Die gordische Auflösung von Knoten, Math. Z. 42 (1937) 680-696 MR1545700

Institute of Mathematics, University of Warsaw

ul. Banacha 2, 02-097 Warszawa, Poland

Fakultät für Mathematik, Universität Regensburg

D-93053 Regensburg, Germany

mcboro@mimuw.edu.pl, sfriedl@gmail.com

www . mimuw. edu.pl/ mcboro,

www . uni-regensburg.de/Fakultaeten/nat_Fak_I/friedl/

Received: 30 November 2012 Revised: 18 June 2014 
